\title{
Deficiency of osteoblastic Arl6ip5 impaired osteoblast differentiation and enhanced osteoclastogenesis via disturbance of ER calcium homeostasis and induction of ER stress-mediated apoptosis
}

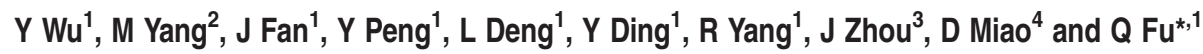

\begin{abstract}
ADP-ribosylation-like factor 6 interacting protein 5 (Arl6ip5), which belongs to the prenylated rab-acceptor-family, has an important role in exocytic protein trafficking, glutathione metabolism and involves in cancer progression. However, its expression pattern and functional role in bone are unknown. Here we demonstrate that Arl6ip5 knock-out mice (Arl6ip5 ${ }^{\Delta 2 / \Delta 2}$ ) show marked decrease of bone mineral density, trabecular bone volume and trabecular thickness. Histomorphometric studies reveal that bone formation parameters are decreased but bone resorption parameters and mRNA level of osteoclast-specific markers are increased in Arl6ip $5^{\Delta / \Delta 2}$ mice. In osteoblast, we demonstrate that Arl6ip5 abundantly expresses in osteoblastic cells and is regulated by bone metabolism-related hormones and growth factors. In vitro analysis reveals that osteoblast proliferation and differentiation are impaired in Arl6ip5 knocked-down and deficient primary osteoblast. Arl6ip5 is also found to function as an ER calcium regulator and control calmodulin signaling for osteoblast proliferation. Moreover, Arl6ip5 insufficiency in osteoblast induces ER stress and enhances ER stress-mediated apoptosis. CCAAT/enhancer-binding protein homologous protein (Chop) is involved in the regulation of apoptosis and differentiation in Arl6ip5 knocked-down osteoblasts. For osteoclastogenesis, Arl6ip5 insufficiency in osteoclast precursors has no effect on osteoclast formation. However, knocked-down osteoblastic Arl6ip5 induces receptor activator of nuclear factor- $\kappa B$ ligand (RANKL) expression and enhances osteoclastogenesis. In addition, ER stress and Chop are involved in the RANKL expression in Arl6ip5 knocked-down osteoblasts. In conclusion, we demonstrate that Arl6ip5 is a novel regulator of bone formation in osteoblasts.
\end{abstract}

Cell Death and Disease (2014) 5, e1464; doi:10.1038/cddis.2014.427; published online 16 October 2014

Bone is a dynamic tissue that undergoes constant remodeling throughout life. ${ }^{1}$ Bone remodeling is a complex process involving the removal of mineralized bone by osteoclasts and the formation of bone matrix through osteoblasts. Osteoclasts, derived from hematopoietic stem cells, control bone resorption. Two cytokines, receptor activator of nuclear factor-kB ligand (RANKL) and macrophage colony-stimulating factor (M-CSF), are necessary for the proliferation and differentiation of osteoclast precursors, as well as the maintenance of survival and activation of osteoclasts. ${ }^{1}$ Mature osteoblasts are differentiated from mesenchymal progenitors via a set of distinct cellular intermediates. ${ }^{2}$ The process of cellular differentiation from mesenchymal progenitors to osteoblast is modulated by a network of signaling pathways and Runx2 and osterix transcription factors. ${ }^{2,3}$ Osteoblast proliferation, survival and differentiation are also regulated by some intracellular events, such as calcium signaling and unfolded protein response (UPR). ${ }^{4-6}$

Calcium $\left(\mathrm{Ca}^{2+}\right)$ is an essential intracellular signaling molecule involved in the regulation of numerous cellular processes including cell proliferation, differentiation, morphology and function. ${ }^{7}$ In resting cell, the concentration of intracellular $\mathrm{Ca}^{2+}\left(\left[\mathrm{Ca}^{2+}\right]_{\mathrm{i}}\right)$ is maintained in very low level and the cytoplasmic $\mathrm{Ca}^{2+}$ is actively pumped from cytosol into endoplasmic reticulum (ER), an intracellular $\mathrm{Ca}^{2+}$ storage organelle, or extruded by $\mathrm{Ca}^{2+}$ transport systems to the extracellular space. $\left[\mathrm{Ca}^{2+}\right]_{i}$ can be suddenly raised via $\mathrm{Ca}^{2+}$ influx from the extracellular space or $\mathrm{Ca}^{2+}$ release from ER. ${ }^{8}$ The release of $\mathrm{Ca}^{2+}$ from $\mathrm{ER}$ is mainly regulated by the inositol trisphosphate 3 (IP3) receptors (IP3Rs) in unexcitable cells

\footnotetext{
${ }^{1}$ Key Laboratory of Nuclear Medicine, Ministry of Health, Jiangsu Key Laboratory of Molecular Nuclear Medicine, Jiangsu Institute of Nuclear Medicine, Wuxi, China; ${ }^{2}$ Department of Radiotherapy, The First Affiliated Hospital of Nanjing Medical University, Nanjing, China; ${ }^{3}$ Department of Molecular Cell Biology and Toxicology, School of Public Health, Nanjing Medical University, Nanjing, China and ${ }^{4}$ State Key Laboratory of Reproductive Medicine, The Research Center for Bone and Stem Cells, Department of Anatomy, Histology, and Embryology, Nanjing, China

${ }^{*}$ Corresponding author: Q Fu, Key Laboratory of Nuclear Medicine, Ministry of Health, Jiangsu Key Laboratory of Molecular Nuclear Medicine, Jiangsu Institute of Nuclear Medicine, Wuxi, Jiangsu 214063, China. Tel: +86 0510 85508776; Fax: +86 0510 85508775; E-mail: fuqiang @jsinm.org

Abbreviations: 2-APB, 2-aminoethoxydiphenyl borate; 4-PBA, 4-phenylbutyric acid; ALP, alkaline phosphatase; Arl6ip5, ADP-ribosylation-like factor 6 interacting protein 5; ATF4, activating transcription factor 4; BMP-2, bone morphogenetic protein 2; CaM, calmodulin; CaMKII, Ca2+/calmodulin-dependent protein kinase Il; Chop, CCAAT/Enhancer-binding protein homologous protein; Dex, dexamethasone; ER, endoplasmic reticulum; IP3Rs, inositol trisphosphate 3 receptors; ISO, isoproterenol hydrochloride; MAR, mineral apposition rate; POBs, primary calvarial osteoblasts; PTH, parathyroid hormone; RANKL, receptor activator of nuclear factor- $\kappa$ B ligand; TG, thapsigargin; TGF $\beta$, transforming growth factor $\beta$; TRAP, tartrate resistant acid phosphatase; UPR, unfolded protein response; $\mu$-CT, micro-computed tomography Received 04.6.14; revised 29.8.14; accepted 04.9.14; Edited by M Agostini
} 
and ryanodine receptors (RyRs) in excitable cells. ${ }^{9}$ Calcium activates signaling pathway via its binding protein calmodulin (CaM) and downstream kinases. The CaM-CaMKII $\left(\mathrm{Ca}^{2}\right.$ ${ }^{+}$/CaM-dependent protein kinase II, CaMKII) pathway has been demonstrated to regulate osteoblast proliferation and differentiation. ${ }^{4,10}$ In addition, $\mathrm{Ca}^{2+}$ is also involved in the synthesis, folding and post-translational modifications of proteins in ER. Disturbance of $\mathrm{Ca}^{2+}$ balance activates UPR that attempts to restore the homeostasis. ${ }^{11}$

UPR, with its ability to sense the insufficiency of protein folding in ER and communicate this information to gene expression programs, has critical roles in the establishment and maintenance for cellular homeostasis, especially in highly secreting cells, such as osteoblasts that produce many important factors for bone formation and bone resorption. ${ }^{6,12}$ Usually, the unfolded protein stress in ER (ER stress) activates the ATF6, IRE1 and PERK - branches of UPR and regulates the expression of target genes involved in the modulation of ER protein folding, such as Bip, Grp94 and XBP1. ${ }^{12}$ The deficiency of UPR signaling proteins such as activating transcription factor 4 (ATF4) and OASIS lead to aberrant bone development and bone loss phenotype in mice. ${ }^{13,14}$ It has been demonstrated that mild ER stress is helpful for osteoblast differentiation. ${ }^{5}$ However, if the stress prolonged and unmitigated, the UPR switches over to initiate cell apoptosis, which is largely mediated by the ATF4-CCAAT/enhancer-binding protein homologous protein (CHOP)-GADD34 signaling axis. ${ }^{15,16}$ It is still not clear whether this persistent stress in osteoblast would lead to apoptosis and affect the communication between osteoblast and other bone cells such as osteoclast.

ADP-ribosylation-like factor 6 interacting protein 5 (Arl6ip5, synonym JWA), which belongs to the prenylated rab-acceptorfamily, ubiquitously expresses in various tissues and is induced by diversity of stimuli such as ER $\mathrm{Ca}^{2+}$-depletion, heat shock and oxidative stress. ${ }^{17-19}$ A microarray study demonstrated that the Arl6ip5 is one of the genes regulated by the parathyroid hormone (PTH) and PTHrP in osteoblasts. ${ }^{20}$ In our previous study, we constructed Arl6ip5 conditional knockout (KO) mice and found some bone-related phenotypes such as kyphosis, ${ }^{21}$ but its exact role in bone metabolism and the underlying mechanism are largely unknown.

In this study, we investigate the role of Arl6ip5 in bone with an exquisite analysis for the bone metabolism-related parameters and find that Arl6ip5 $\mathrm{KO}$ in mice lead to bone loss. We also find that Arl6ip5 is an ER localized protein in osteoblast and is regulated by osteotropic factors. Arl6ip5 insufficiency in osteoblast disturbs calcium homeostasis, induced ER stressmediated apoptosis and impairs osteoblast proliferation and differentiation. Moreover, Arl6ip5 insufficiency indirectly enhances the osteoclastogenesis through increasing RANKL expression in osteoblast.

\section{Results}

Arl6ip5 deficiency induces bone loss phenotype in mice. Arl6ip5 is a widely expressed protein. Here, we found that Arl6ip5 mRNA also expressed in bone tissues lumbar vertebra, tibia, femur and calvaria (Supplementary Figure S1).
To assess the in vivo role of Arl6ip5, we constructed the Arl6ip5 deficiency mice with Arl6ip5 exon2 deletion in whole body (Arl6ip5 $5^{\Delta 2 / \Delta 2}$ mice) $)^{21}$ and found these mice with growth retardation and severe scoliosis, which were not observed in Arl6ip5 $^{+/+}$mice. The micro-computed tomography ( $\mu$-CT) analysis showed that the bone mineral density of tibia and vertebra were $10-15 \%$ reduced in Arl6ip $5^{\Delta 2 / \Delta 2}$ mice compared with control littermates at 4 months of age (Figure 1a and Supplementary Figure S2), which was observed in both genders (data not shown). Quantitative analyses further demonstrated that $40 \%$ less of BV/TV $(P<0.05)$ and significant decrease of trabecular thickness (Tb.Th) $(P<0.05)$ and trabecular number (Tb.N) $(P<0.05)$ but significant increase of trabecular separation (Tb.Sp) $(P<0.05)$ in Arl6ip5 ${ }^{\Delta 2 / \Delta 2}$ mice compared with Arl6ip5 $5^{+/+}$mice (Figure 1a). However, no distinction was found in levels of serum calcium, phosphate, glucose, albumin and cholesterol between Arl6ip5 $5^{\Delta 2 / \Delta 2}$ mice and Arl6ip5 $5^{+/+}$mice (data not shown).

Dynamic histomorphometry of the proximal tibia revealed that bone formation parameters such as mineral apposition rate (MAR, $P<0.01)$, BFR/BS $(P<0.01)$ and $\mathrm{dLS} / \mathrm{BS}$ $(P<0.01)$ of cortical bone (Figure $1 \mathrm{~b})$ and MAR of trabecular bone (Supplementary Figure S3) were significantly decreased in Arl6ip5 $5^{\Delta / \Delta 2}$ mice compared with control mice at 4 months of age. Histological analysis further revealed a significant decrease in osteoblasts number $(P<0.05)$ (Figure $1 \mathrm{~b})$ and an increase in the number of tartrate-resistant acid phosphatase (TRAP)-positive osteoclasts $(P<0.05)$ in the proximal tibia of Arl6ip5 $5^{\Delta 2 / \Delta 2}$ mice compared with Arl6ip5 ${ }^{+/+}$mice (Figure 1c). In consistence, the serum level of cTX-II (Figure 1d) and mRNA expression of RANKL (1.49-fold, $P<0.05)$, Trap (3.35-fold, $P<0.05)$ and Ctsk $(3.45$-fold, $P<0.05$ ) (Figure 1e) in the tibia of $A r l 6 i p 5^{\Delta 2 / \Delta 2}$ mice were also significant higher than that in control mice.

Arl6ip5 localizes in ER and is stimulated by osteotropic factors in osteoblast. To understand the role of Arl6ip5 in osteoblasts, the mRNA level and subcellular localization of Arl6ip5 were determined in primary calvarial osteoblasts (POBs) and stromal/osteoblast cell line (UAMS-32). We found that Arl6ip5 mRNA expressed in bone marrow cells, POBs and osteoblast cell line (data not shown). For bone marrow cells, the mRNA level of Arl6ip5 in adherent cells was significantly higher than that in non-adherent cells (Supplementary Figure S4). In the differentiated UAMS-32 cells induced by bone morphogenetic protein 2 (BMP-2), as identified by the enhancing expression of specific osteoblast differentiation markers alkaline phosphatase (ALP) and Col1a1, the expression of Arl6ip5 was increased (Figures $2 a-c)$. In UAMS-32 cells, the expression of Arl6ip5 was quickly upregulated by osteotropic factors (Figure $2 \mathrm{~d}$ ). The peak level of Arl6ip5 expression was at $3 \mathrm{~h}$ for dexamethasone (Dex) treatment (3.83-fold, $P<0.05)$ and at $6 \mathrm{~h}$ for transforming growth factor $\beta 1$ (TGF- $\beta 1$; 2.34-fold, $P<0.05$ ), BMP-2 (2.41-fold, $P<0.05)$ and PTH treatment $(2.33-$ fold, $P<0.01)$, respectively. The expression returned to basic level at $24 \mathrm{~h}$ after treatment, except for treatment with BMP-2 (1.69-fold, $\quad P<0.05)$ and PTH $(1.76$-fold, $\quad P<0.05)$ (Figure $2 \mathrm{~d}$ ). For the subcellular localization of Arl6ip5 protein 
a

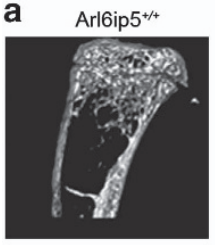

b
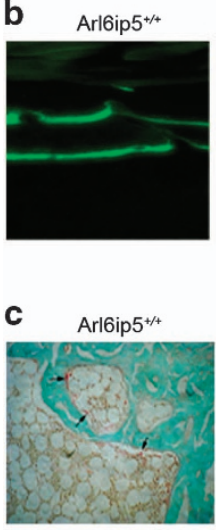

Arl6ip5 $5^{\Delta / \Delta 2}$

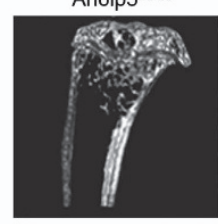

Arl6ip5 $5^{f \Delta / \Delta 2}$
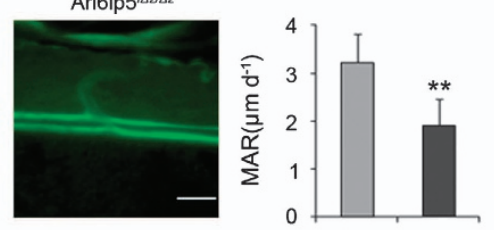

Arl6ip5 $5^{\Delta / \Delta 2}$

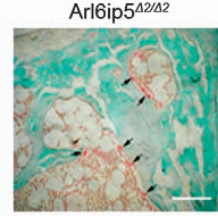

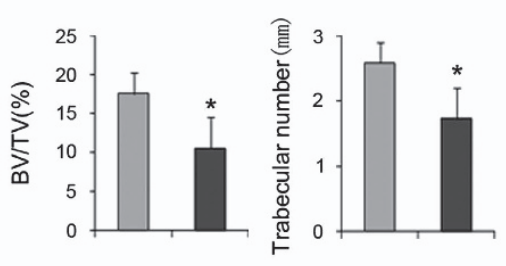
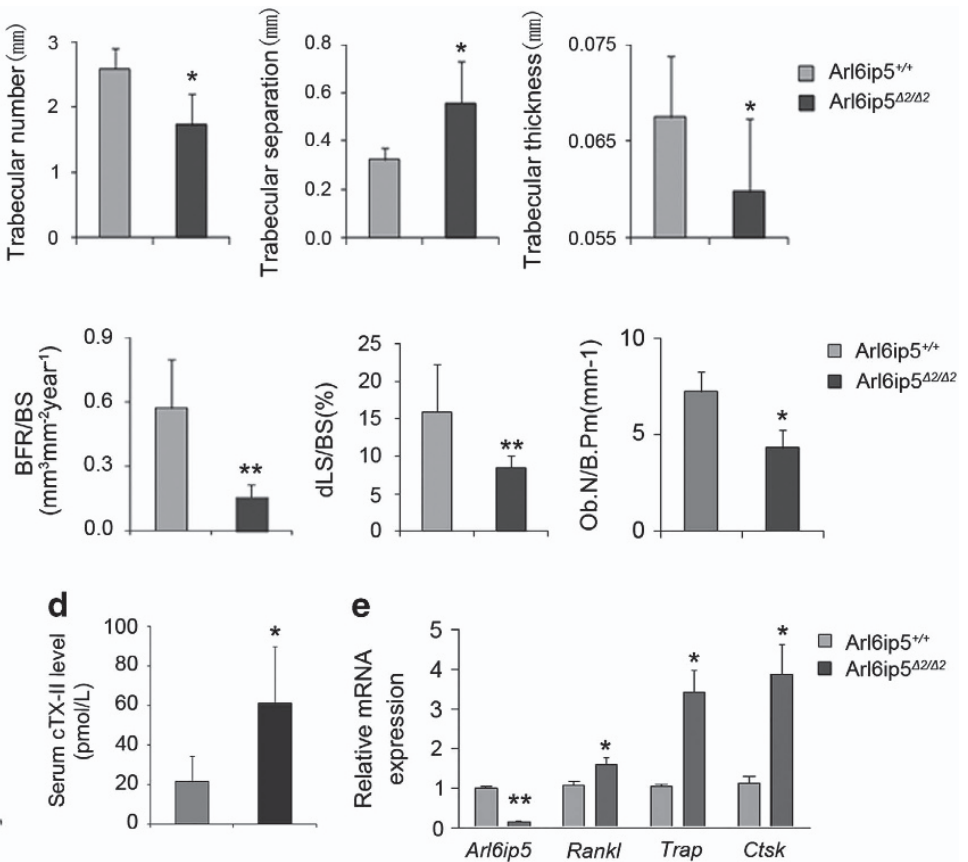

Figure 1 Arl6ip $5^{\Delta 2 / \Delta 2}$ mice show bone loss phenotype. (a) $\mu$-CT images and three-dimensional microstructural analysis of tibias from 16-week-old Arl6ip5 ${ }^{+/+}(n=10)$,

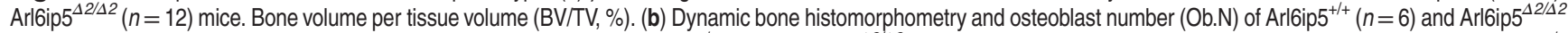
mice $(n=6)$. (c) TRAP staining images of the tibias from 16-week-old Arl6ip5 $5^{+/+}(n=6)$, Arl6ip $5^{\Delta 2 \Delta 2}(n=6)$ mice. Scale bar, $100 \mu$ m. (d) The serum level of cTx-II in Arl6ip5 ${ }^{+/+}$ $(n=6)$ and Arl6ip5 $5^{\Delta 2 / \Delta 2}$ mice $(n=6)$. (e) Expression of Arl6ip5, RANKL, Trap and CtskmRNAs in the tibias from Arl6ip5 $5^{\Delta 2 / \Delta 2}(n=6)$ and Arl6ip5 $5^{+/+}(n=6)$ mice. In all panels, bar represents mean \pm S.E.M., ${ }^{*} P<0.05,{ }^{* *} P<0.01$. All $P$-values were based on Students $t$-test

a

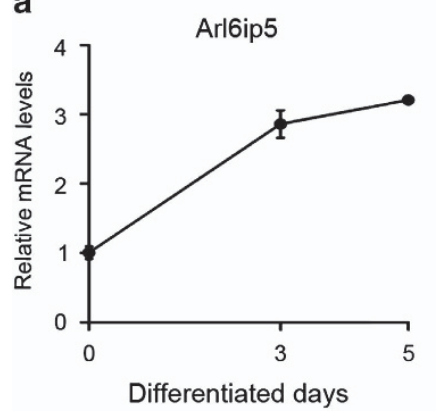

d

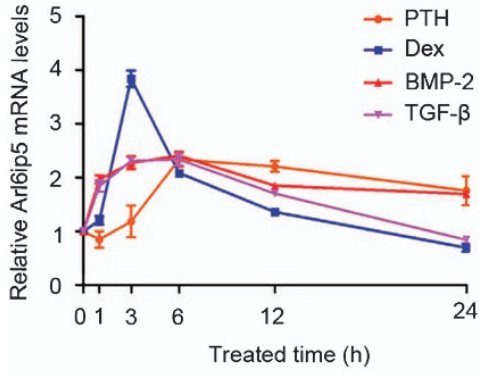

b

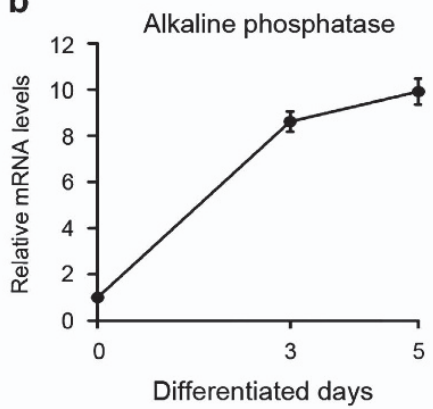

c

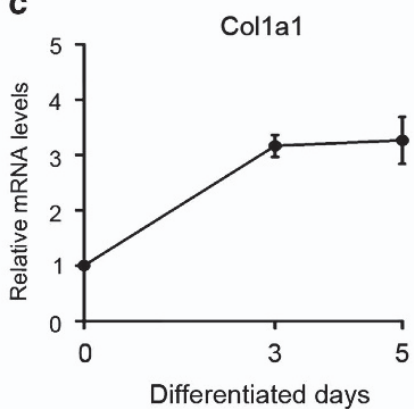

e

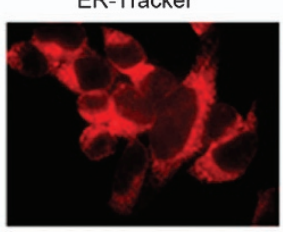

Calnexin

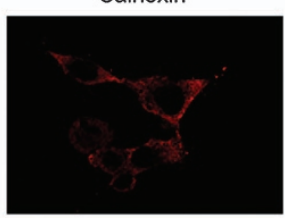

Arl6ip5-EGFP

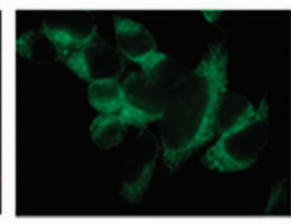

Arl6ip5

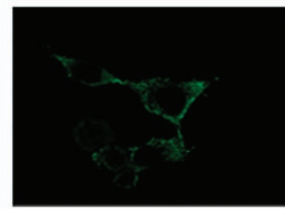

Merge

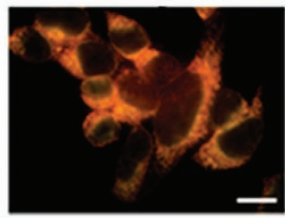

Merge

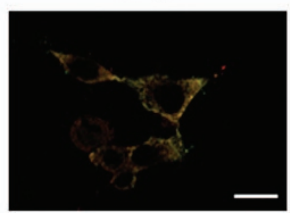

Figure 2 The basic and induced expression pattern of Arl6ip5 in osteoblasts. (a-c) UAMS-32 cells were treated with BMP-2 (100 ng/ml) for the indicated days to induce differentiation, then mRNA level of Arl6ip5 (a), alkaline phosphatase (b) and Col1a1(c) was analyzed with Q-PCR. $n=3$. In all panels, bars represent mean \pm S.E.M. (d) Arl6ip5 mRNA levels detected by Q-PCR analysis after treating UAMS-32 cells with indicated dosage of PTH (100 nM), Dex (400 nM), TGF $\beta 1$ ( $10 \mathrm{ng} / \mathrm{ml})$ and BMP-2 (100 ng/ml) for 0,1 , 3, 6, 12 and 24 h. $n=4$. (e) Arl6ip5-EGFP was transiently transfected into POBs and its localization with ER-specific indicator (ER-tracker) was analyzed with fluorescence microscope (upper panel). Immunofluorescence staining with the antibodies to Arl6ip5 and ER marker calnexin was used to analyze the localization of endogenous Arl6ip5 to ER (lower panel). Scale bar $=10 \mu \mathrm{m}$ 
in osteoblasts, we observed that both exogenous Arl6ip5EGFP protein and endogenous Arl6ip5 was highly overlapped with the ER as traced by ER-tracker and protein calnexin in POBs (Figure 2e) and in UAMS-32 cells (data not shown). In addition, endogenous Arl6ip5 was not immunolocalized to Golgi body as shown by the coimmunostaining with the Golgi protein GM130 (data not shown).

\section{Arl6ip5 regulates osteoblast proliferation and differentiation.} To further understand the possible function of Arl6ip5 in osteoblast, a verified small interfering RNA (siRNA) (Supplementary Figure S5) was used to knock down Arl6ip5 expression in UAMS-32 cells. The 3-(4,5-dimethylthiazol-2yl)-2,5-diphenyltetrazolium bromide (MTT) assay displayed that silence of Arl6ip5 reduced cell proliferation (Figure 3a), which was also observed in Arl6ip5 ${ }^{\Delta 2 / \Delta 2}$ POBs when compared with Arl6ip5 ${ }^{+/+}$POBs (Figure $3 b$ ). On the contrary, overexpression of Arl6ip5 in UAMS-32 cells with HA-tagged mouse Arl6ip5 (HA-Arl6ip5) significantly increased cell proliferation (Figure $3 c$ ). For osteoblast differentiation, the ALP-positive cells and the ALP activity in cultured Arl6ip5 $5^{+/+}$ POBs were increased in time-dependent manner, but were just slightly changed in cultured Arl6ip5 $5^{\Delta 2 / \Delta 2}$ POBs (Figures $3 d$ and $e$ ). The expression of osteoblastic differentiation markers, ALP, Runx2, osterix, osteocalcin and osteopontin in Arl6ip5 $5^{\Delta 2 / \Delta 2}$ POBs were also relatively lower compared with Arl6ip5 ${ }^{+/+}$POBs (Figures $3 \mathrm{f}-\mathrm{k}$ ).

Arl6ip5 regulates ER calcium and activated CaM pathway. The homeostasis of intracellular $\mathrm{Ca}^{2+}$ level $\left(\left[\mathrm{Ca}^{2+}\right]_{\mathrm{i}}\right)$,
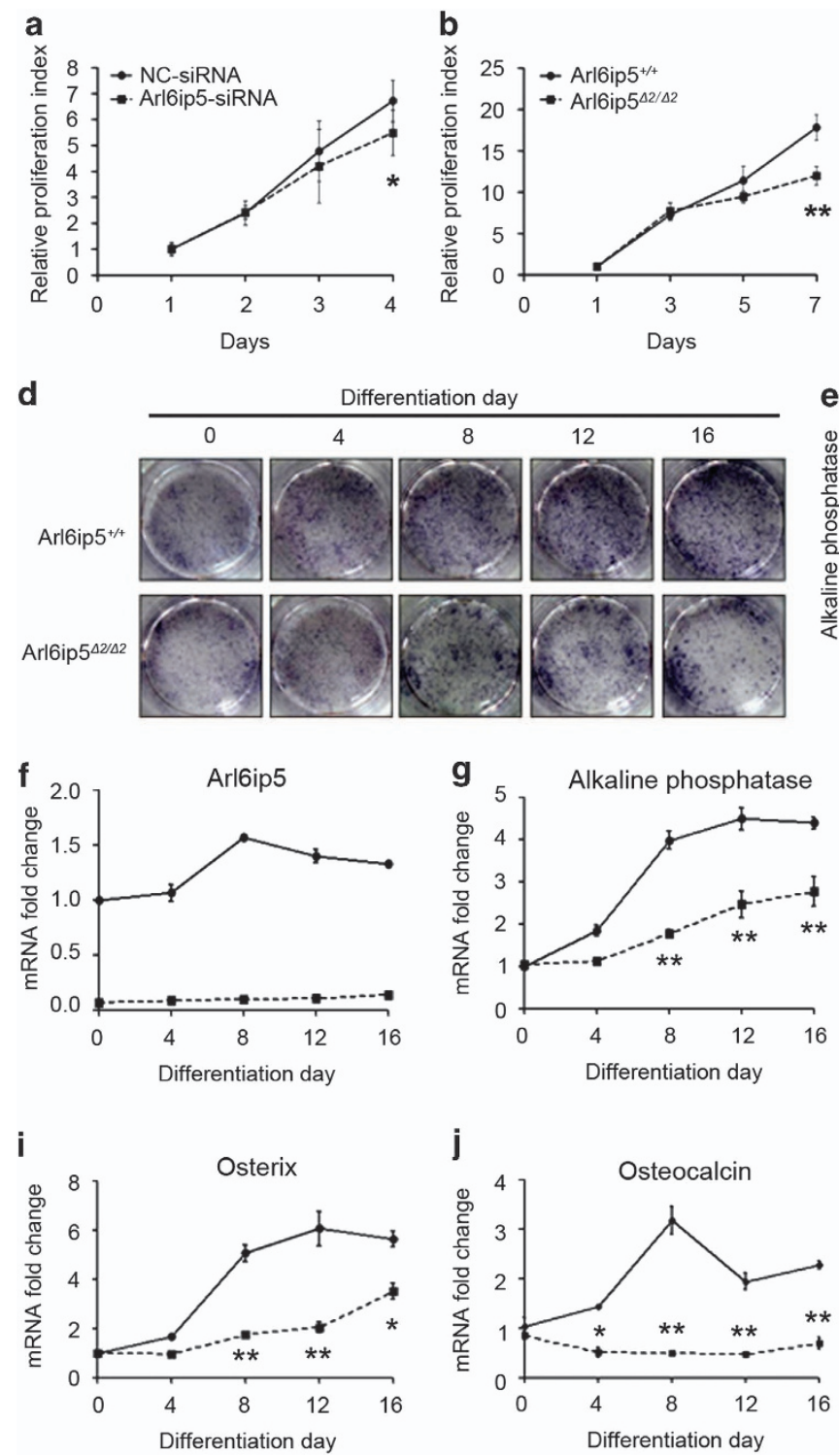
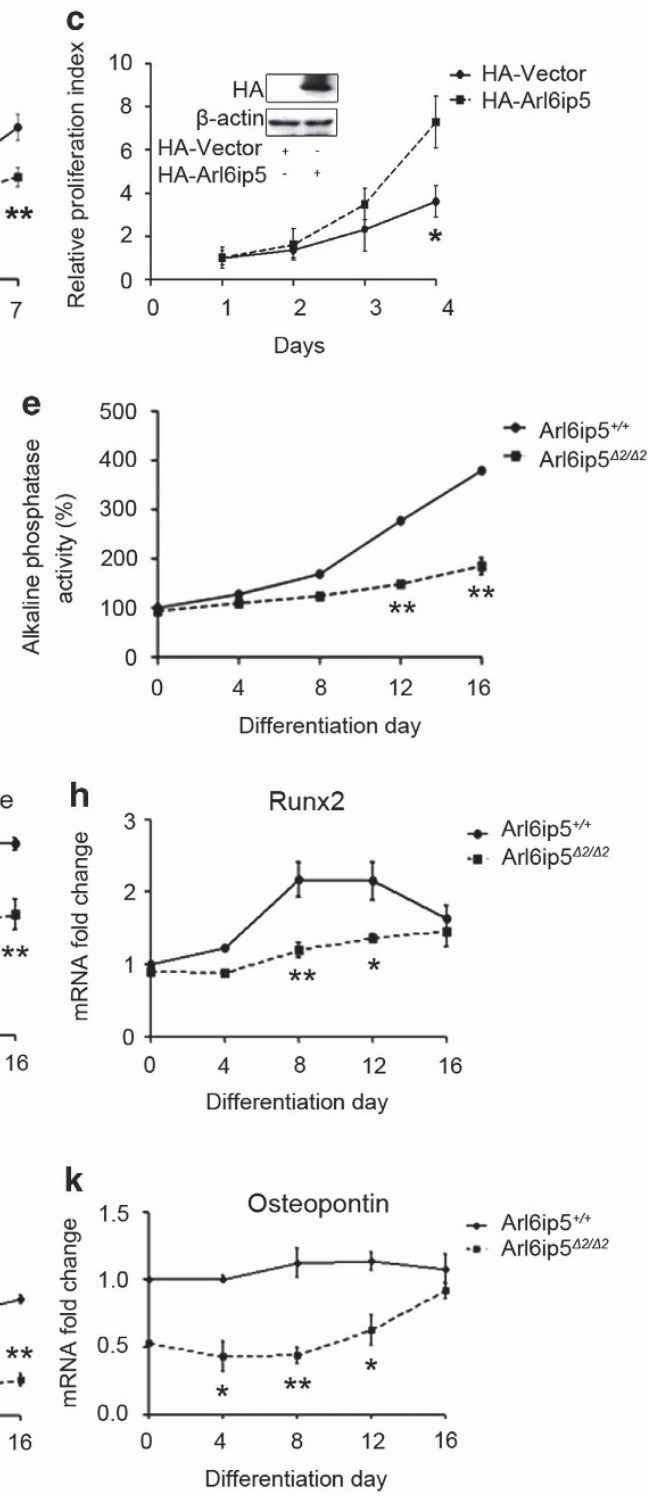

Figure 3 Arl6ip5 affects osteoblast proliferation and differentiation. Cell proliferation in UAMS-32 cells with Arl6ip5-siRNA (a) and HA-tagged Arl6ip5 (c) treatments were analyzed with MTT assay. The proliferation rate between Arl6ip5 ${ }^{+/+}$and Arl6ip5 ${ }^{\Delta 2 / \Delta 2}$ POBs (b) was also compared. Arl6ip5 ${ }^{+/+}$and Arl6ip5 ${ }^{\Delta 2 / \Delta 2}$ POBs were cultured in differentiated medium and evaluated for ALP staining (d), ALP activity (e) and mRNA levels of Arl6ip5, ALP, Runx2, osterix, osteocalcin and osteopontin ( $\mathbf{f}-\mathbf{k})$. ${ }^{*} P<0.05$; ${ }^{* \star} P<0.01$ by Students $t$-test. In all panels except $\mathbf{d}, n=3$. In $\mathbf{d}$, representative results were shown from three independent experiments 
which could be modulated by some ER localized proteins, is important for osteoblast differentiation. ${ }^{4,22}$ Arl6ip5 was an ERresident protein in osteoblast and could be evoked by $\mathrm{Ca}^{2+}$ deletion, ${ }^{19}$ therefore, we evaluated whether this protein was also involved in the regulation for $\left[\mathrm{Ca}^{2+}\right]_{i}$ in osteoblasts. Our results indicated that ATP stimulated $\left[\mathrm{Ca}^{2+}\right]_{i}$ were decreased in Arl6ip5 knocked-down cells and in Arl6ip5 ${ }^{\Delta 2 / \Delta 2}$ POBs (Figure 4a and Supplementary Figure S6) but increased in Arl6ip5overexpressed UAMS-32 cells (Figure 4b) compared with respective controls. Moreover, in BMP-2-treated UAMS-32 cells, silence of Arl6ip5 decreased but overexpression of Arl6ip5 increased $\left[\mathrm{Ca}^{2+}\right]_{i}$ level (Figures $4 \mathrm{c}$ and $\mathrm{d}$ ). By measuring the cytosolic $\mathrm{Ca}^{2+}$ peak, $^{23}$ we found that Arl6ip5 deficiency decreased $\mathrm{ER} \mathrm{Ca}^{2+}$ store in POBs and Arl6ip5 stable overexpression led to a significant increase in $\mathrm{ER} \mathrm{Ca}^{2+}$ levels in UAMS-32 cells (Supplementary Figure S7).

Intracellular $\mathrm{Ca}^{2+}$ activates and affects many signaling pathways that modulate cell differentiation, such as CaMCaMKII-NFATc1 pathway. ${ }^{4}$ Despite the CaM protein level was not changed, the phosphorylated CaMKII was decreased in Arl6ip5 knocked-down cells and increased in Arl6ip5 overexpressed cells (Figure 4e). IP3Rs rather than RyRs were expressed in UAMS-32 cells (data not shown). To further analyze the activation mechanism of Arl6ip5 on $\mathrm{Ca}^{2+}$ channel, treated cells with 2-aminoethoxydiphenyl borate (2-APB), the inhibitor of IP3Rs ${ }^{24}$ completely blocked $\mathrm{Ca}^{2+}$ release from the IP3R channel in control cells but partially reduced the $\left[\mathrm{Ca}^{2+}\right]_{i}$ levels in Arl6ip5 stable overexpressed UAMS-32 cells (Figure 4f). However, thapsigargin (TG), an ATPase inhibitor for SERCA ${ }^{25}$ completely inhibited $\mathrm{Ca}^{2+}$ release from the ER in both controls and Arl6ip5 stable overexpressed cells (Figure 4e).

\begin{abstract}
Arl6ip5 deficiency activates ER stress-mediated apoptosis in osteoblast. The disturbance of ER calcium homeostasis leads to accumulation of unfolded proteins in ER, thereby initiating ER stress and activating UPR. ${ }^{11}$ Immunostaining showed that the bone matrix proteins, such as procollagen-1 and osteocalcin (Ocn), were accumulated in ER of Arl6ip5 ${ }^{\Delta 2 / \Delta 2}$ POBs but not in Arl6ip5 ${ }^{+/+}$POBs (Figure 5a). UPR target genes P4hb, Grp94 and Pdia3, which help in relieving ER stress, and the genes Chop and Gadd34, which mediate pro-apoptosis signal of ER stress, ${ }^{12}$ were significantly increased in Arl6ip5 $5^{\Delta 2 / \Delta 2}$ POBs compared with Arl6ip5 $^{+/+}$(Figure 5b). Moreover, the mRNA levels of Chop and Gadd34 were also increased significantly in differentiated Arl6ip5 ${ }^{\Delta 2 / \Delta 2}$ POBs compared with Arl6ip5 ${ }^{+/+}$POBs (Figure 5b). However, the XBP1s (spliced form of XBP1) was not detected, even in differentiated Arl6ip5 ${ }^{\Delta 2 / \Delta 2}$ POBs and Arl6ip5 ${ }^{+/+}$POBs (data not shown). The expression of Chop and Gadd34 genes were also increased in the tibia extracts from Arl6ip5 ${ }^{\Delta 2 / \Delta 2}$ mice (Supplementary Figure S8) and in Arl6ip5 knocked-down UAMS-32 cells (Supplementary Figure S9). BMP-2 treatment slightly enhanced the expression of Bip, Chop and Gadd34 in UAMS-32 cells, but the enhancement was obviously raised after Arl6ip5 was silenced (Figure 5c).
\end{abstract}

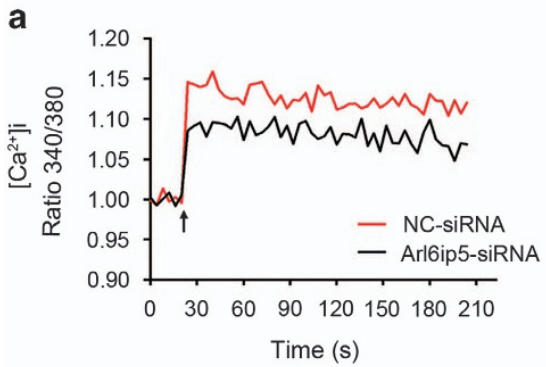

d

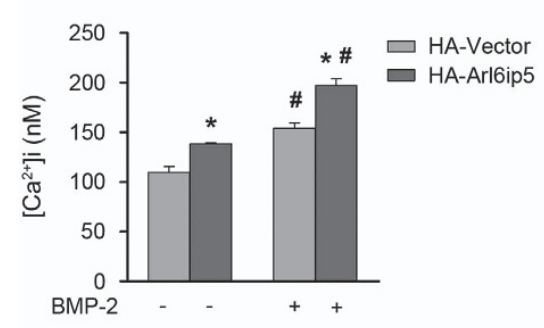

b
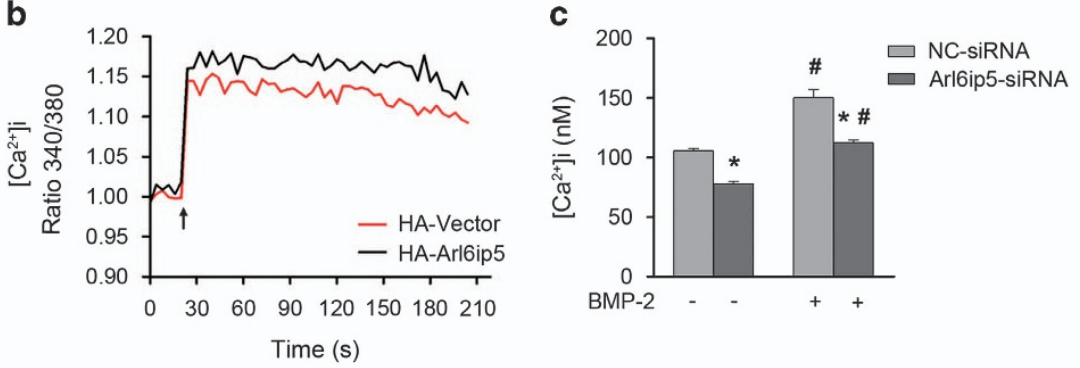

e

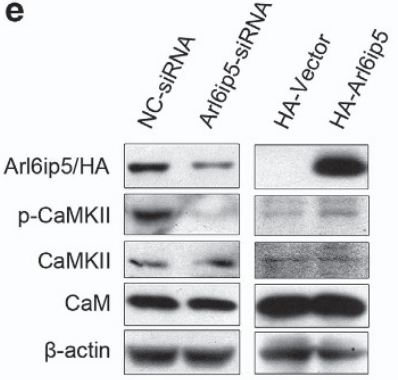

f

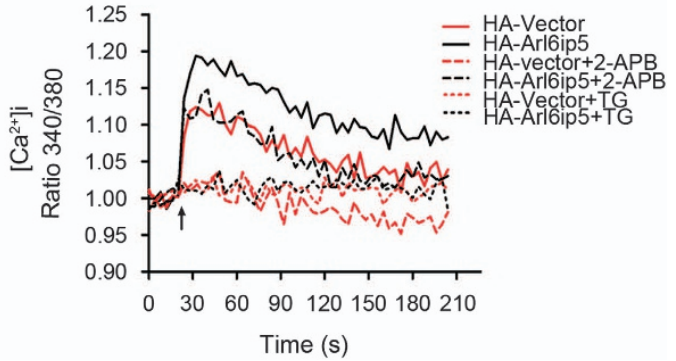

Figure 4 Arl6ip5 regulates intracellular calcium level and $\mathrm{Ca}^{2+}$-CaM signaling. ATP-stimulated $\left[\mathrm{Ca}^{2+}\right]_{\mathrm{i}}$ in a time course was analyzed in UAMS-32 cells received Arl6ip5-siRNA (black) and its negative control (NC-siRNA) (red) for $72 \mathrm{~h}$ (a) and in UAMS-32 cells stably transfected with pcDNA3.1(+) (HA-vector) (red) or HA-Arl6ip5 (black) (b). In a and b, the data shown are representative of at least four different experiments. (c and d) $\left[\mathrm{Ca}^{2+}\right]_{i}$ levels in BMP-2-treated UAMS-32 cells. Arl6ip5-siRNA and NC-siRNA treated (72 $\mathrm{h}$ ) or HAVector and HA-Arl6ip5 stably expressed UAMS-32 cells were cultured with BMP-2 (100 ng/ml) for $6 \mathrm{~h}$. *HA-Arl6ip5 or Arl6ip5-siRNA versus control, $P<0.05$; ${ }^{*} \mathrm{BMP}-2$ treatment versus no treatment, $P<0.05$. Error bars represent the mean \pm S.E.M., $n=3$. (e) Arl6ip5 activates the $\mathrm{Ca}^{2+}$-CaM signaling pathways. In UAMS-32 cells with HA-Arl6ip5 stably overexpressed or Arl6ip5-siRNA transiently transfected, the levels of the signal molecules were analyzed by western blotting. (f) UAMS-32 cells stably transfected with HA-Vector or HA-Arl6ip5 were analyzed for ATP-stimulated $\left[\mathrm{Ca}^{2+}\right]_{\mathrm{i}}$. 2-APB $(100 \mu \mathrm{M})$ and TG $(1 \mu \mathrm{M})$ were added to the cell culture for 30 min before ATP stimulation. Representative results were shown from three independent experiments. In panel $\mathbf{a}, \mathbf{b}$ and $\mathbf{f}$, arrows indicated ATP stimulation 
As Chop and Gadd34 mediates the pro-apoptosis signal of UPR. ${ }^{12,16}$ The elevated Chop and Gadd34 expression suggested that apoptosis may be initiated by Arl6ip5 deficiency in osteoblasts. As we suspected, the apoptotic osteoblasts was significantly increased and mRNA level of anti-apoptosis protein $\mathrm{Bcl}-2$ was decreased in Arl6ip5 $5^{\Delta 2 / \Delta 2}$ mice tibia when compared with their controls (Supplementary Figure S10). In consistence, the Annexin-V-positive cells (Figure $5 d$ ) and the level of cleaved caspase-3 and caspase12 proteins (Figure 5e) were markedly increased in Arl6ip5 knocked-down UAMS-32 cells. The pro-apoptotic genes Bim-1 and Puma, but not anti-apoptotic gene Bcl-2, were also raised after silence of Arl6ip5 in UAMS-32 cells (Supplementary Figure S11). Moreover, Annexin-V-positive cells (Figure 5f) and cleaved caspase-3 and caspase-12 proteins (Figure $5 \mathrm{~g}$ ) were markedly decreased in Arl6ip5 knocked-down cells by treatment with 4-phenylbutyrate acid
(4-PBA), a chemical chaperon that prevents protein aggregation and relieves ER stress.

IRE1 and Chop involves in the ER stress-mediated apoptosis. ${ }^{16}$ Although the activity of IRE1 was not changed (Figure 6a), the protein expression of Chop was markedly increased in Arl6ip5 knocked-down cells compared with controls (Figure 6a). The mRNA levels of Trib3, one downstream target gene of Chop, ${ }^{26}$ was also increased (Supplementary Figure S12). Silence of Chop by siRNA (Figure 6b), apparently decreased the expression of Gadd34 and Trib3 (Figures $6 c$ and d), and the number of Annexin-Vpositive cells (Figure 6e) in Arl6ip5 knocked-down cells.

The regulation of Arl6ip5 on osteoblast differentiation and proliferation needs Chop. Chop also involves in the regulation of osteoblast differentiation, and the proliferation in some cells. ${ }^{27-29}$ In this study, knocking down the Chop a

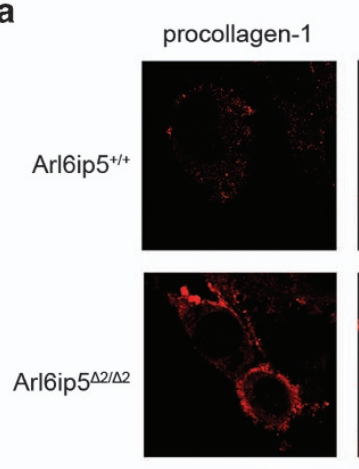

C

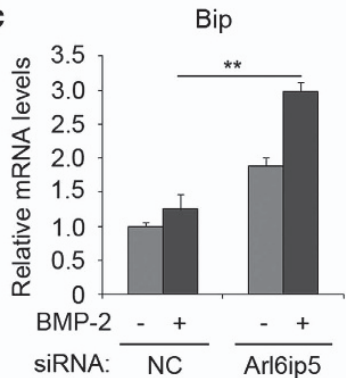

d

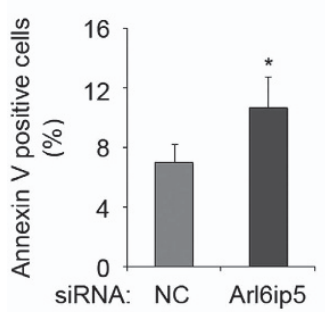

Ocn
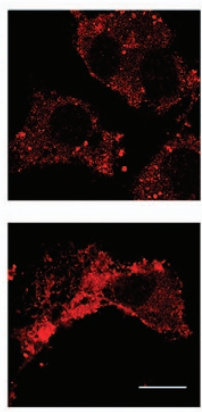

b

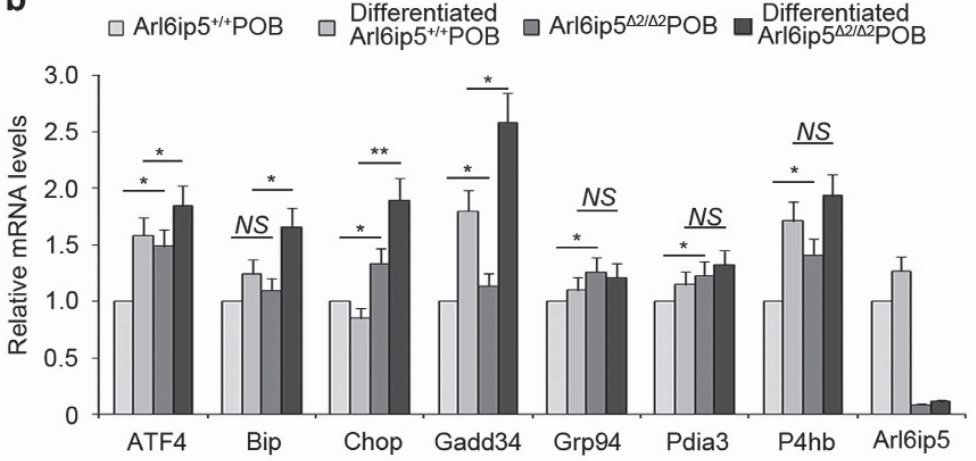

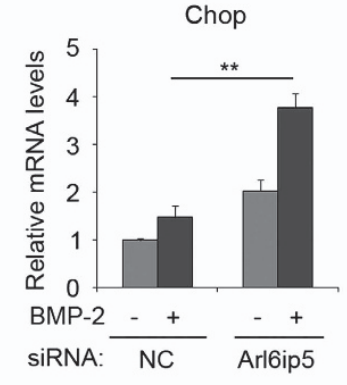

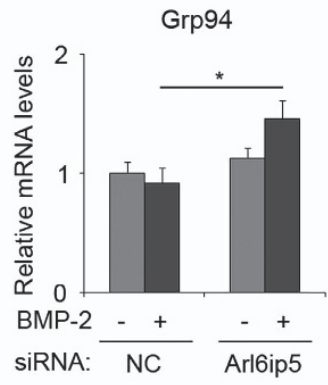

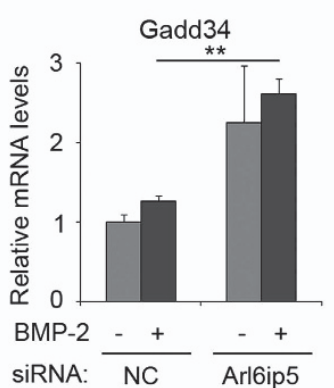

g

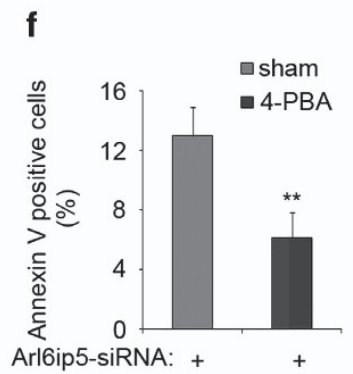

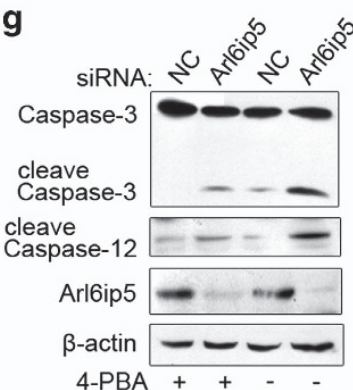

Figure 5 Deficiency of Arl6ip5 in osteoblasts induced ER stress and apoptosis. (a) Immunofluorescence staining using antibodies for procollagen-1 and osteocalcin (Ocn) in Arl6ip5 $5^{+/+}$and Arl6ip5 ${ }^{\Delta 2 / \Delta 2}$ POBs. Scale bar, $10 \mu \mathrm{m}$. (b) Q-PCR analysis was used to detect the mRNA level for ER stress-related genes in POBs of Arl6ip5 $5^{+/+}$and Arl6ip5 $5^{\Delta 2 / \Delta 2}$ without or with differentiated medium treatment. $n=3$. (c) UAMS-32 cells first received NC-siRNA or Arl6ip5-siRNA treatment for $48 \mathrm{~h}$, then received BMP-2 (100 ng/ml) for $24 \mathrm{~h}$. Q-PCR was used to detect the mRNA level for ER stress-related genes. $n=3$. (d and e) The UAMS-32 cells received NC-siRNA or Arl6ip5-siRNA treatment together without (d and $\mathbf{e}$ ) or with 4-PBA $(10 \mathrm{mM}$ ) ( $\mathbf{f}$ and $\mathbf{g}$ ) were stained with Annexin-V and the proportion of Annexin-V-positive cells were calculated ( $\mathbf{d}$ and $\mathbf{f}, n=4)$ or used for immunoblotting for the expression of caspase-3, caspase-12, Arl6ip5 and $\beta$-actin (e and $\mathbf{g}$ ). For the immunoblotting, representative results were shown from three independent experiments. In all panels, error bars represent the mean \pm S.E.M. ${ }^{* *} P<0.01,{ }^{*} P<0.05$ based on ANOVA in $\mathbf{b}$ and $\mathbf{c}$ and Students $t$-test in $\mathbf{d}$ and $\mathbf{f}$ 
a siRNA: NC Arl6ip5
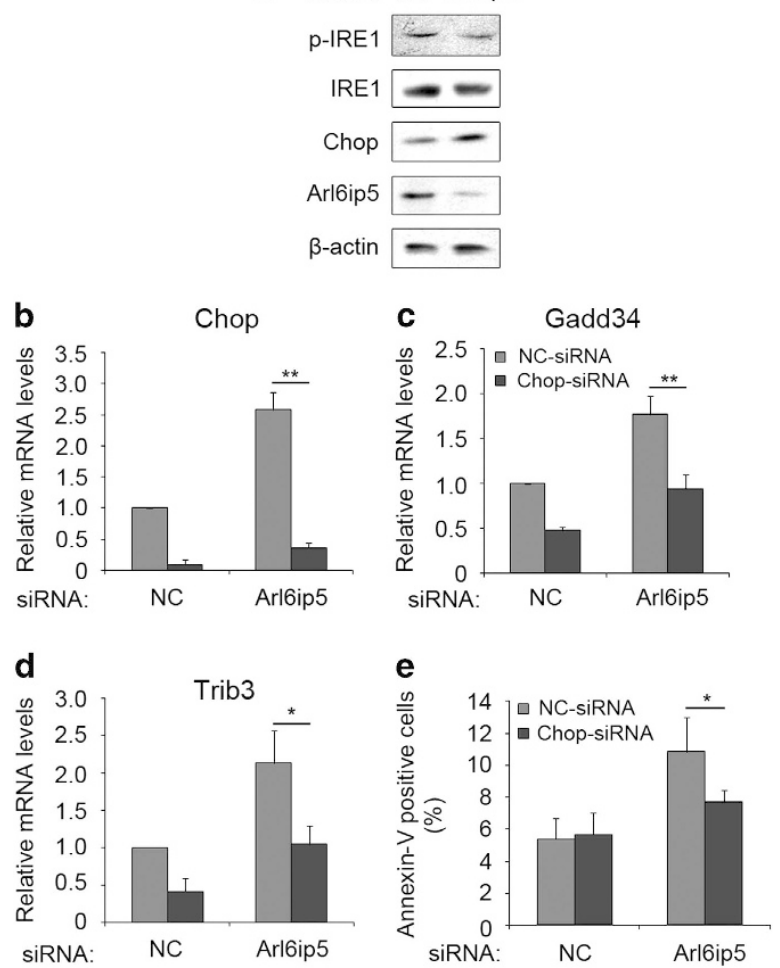

Figure 6 Apoptosis mediated by Arl6ip5 interference is regulated by Chop. (a) Immunoblotting was used to detect the protein level for $p$-IRE1 $\alpha$, IRE1, Chop, Arl6ip5 and $\beta$-actin in the UAMS-32 cell with NC-siRNA or Arl6ip5-siRNA treatment for $72 \mathrm{~h}$. Representative results were shown from three independent experiments. (b-e) UAMS-32 cells first received NC-siRNA or Chop-siRNA for $24 \mathrm{~h}$, then the medium was changed and further received NC-siRNA or Arl6ip5-siRNA for $72 \mathrm{~h}$. Q-PCR was used to analyze the mRNA levels of Chop (b), GADD34 (c) and Trib3 (d). The apoptotic cells were stained with Annexin- $V$ and the proportion were calculated (e). $n=4$. In all panels, error bars represent the mean \pm S.E.M. ${ }^{* *} P<0.01,{ }^{*} P<0.05$ based on ANOVA

significantly increased the ALP-positive cells and ALP activity (Figures $7 \mathrm{a}$ and $\mathrm{b}$ ) and the expression of Runx2 in Arl6ip5siRNA-treated UAMS-32 cells (Figure 7c). For osteoblast proliferation, treating the cells with Chop-siRNA significantly enhanced the expression of c-Fos (Figure 7c), one key transcription factor for cell proliferation, and improved the proliferation ability of Arl6ip5 knocked-down UAMS-32 cells (Figure 7d). Moreover, the proliferation ability of Arl6ip5 deficiency UAMS-32 cells was significantly rescued by the treatment with $\mathrm{CaCl}_{2}$ (Supplementary Figure S13A). The proliferation ability (Figure 7e) and c-Fos mRNA level of HAArl6ip5 overexpressed cells (Supplementary Figure S13B) was inhibited by CaMK kinase inhibitors $\mathrm{KN}-93$ but not STO609 treatments.

Osteoblastic Arl6ip5 regulates RANKL expression. The increase of osteoclast formation in Arl6ip5 ${ }^{\Delta 2 / \Delta 2}$ mice implicated the role of Arl6ip5 in osteoclastogenesis. As markers Trap and Mmp9, Arl6ip5 expression was increased during osteoclast differentiation (Figures 8a-c), however, the TRAPpositive osteoclast formation was comparable between BMMs from wild-type mice and from Arl6ip $5^{\Delta 2 / \Delta 2}$ mice (Figures 8d and e). In consistence, silenced Arl6ip5 expression with siRNA in Raw264.7 cells slightly but not significantly $(P=0.18)$ increased the osteoclast formation when compared with negative control siRNA-treated cells (Figures $8 f$ and $\mathrm{g}$ ). There was also no difference in the expression level of Trap (Figure 8h).

As osteoblast lineage cells (e.g., osteoblast and osteocyte) are coupling with osteoclast and regulate osteoclastogenesis, ${ }^{30}$ it is possible that the increased of osteoclast number is caused by the aberrant expression of coupling factors in Arl6ip5 deficiency osteoblast. To verify this hypothesis, the welldemonstrated coupling factors (CSF-1, RANKL, Sema3a, Sema3b, Sema7a and Wnt5a) that express in osteoblast and regulate osteoclastogenesis were screened for their expression in Arl6ip5 knocked-down UAMS-32 cells. We found that the basic RANKL mRNA was significantly increased (2.3-fold, $P<0.05)$ in Arl6ip5-siRNA-treated UAMS-32 cells (Figure 9a). In consistence, the protein level of RANKL in Arl6ip5-siRNAtreated UAMS-32 cells was increased nearly threefold compared with control siRNA-treated cells (Figure 9b). The RANKL mRNA level in tibia extracts from Arl6ip $5^{\Delta 2 / \Delta 2}$ mice was also significantly higher than in wild-type mice (Figure 1e). Moreover, soluble RANKL (sRNAKL) in serum from Arl6ip $5^{\Delta 2 / \Delta 2}$ mice was nearly twofold higher compared with Arl6ip $5^{+/+}$mice (Figure 9c). Furthermore, the increase of RANKL expression by treatment with PTH, isoproterenol hydrochloride (ISO) or $1,25-(\mathrm{OH})_{2} \mathrm{D}_{3}$ was further increased in Arl6ip5 knocked-down osteoblast (Figures $9 d$ and e and Supplementary Figure S14). The basic and PTH-induced sRANKL level in the culture medium of Arl6ip5-siRNA-treated cells or Arl6ip5 KO POBs were also higher compared with the culture medium of control cells (Supplementary Figure S15). RANKL is essential for osteoclastogenesis. ${ }^{1}$ The numbers of TRAP-positive osteoclast (Figure 9f) and pit formation (Supplementary Figure S16) were much more in the co-culture containing Arl6ip $5^{\Delta 2 / \Delta 2}$ osteoblast than the co-culture containing Arl6ip $5^{+/+}$osteoblast. To further investigate the mechanism for the regulation of Arl6ip5 on RANKL expression, H-89, an inhibitor of PKA activity and 4-PBA, the chemical chaperon as indicated above were used. The induced RANKL expression in Arl6ip5 knocked-down UAMS-32 cells was lower to normal with 4-PBA but not with H-89 treatment (Figure $9 \mathrm{~g}$ and Supplementary Figure S17), however, 4-PBA treatment had no effect on the RANKL expression induced by PTH (Figure 9g). The expression of basic RANKL as Arl6ip5 deficiency was further increased after cells treated with ATF4-siRNA (Supplementary Figure S18), but significantly decreased after Chop gene was interfered (Figure 9h).

\section{Discussion}

The study with gene $\mathrm{KO}$ mice is important to unravel the in vivo functions of one gene. The deletion of exon2 of Arl6ip5 mediated by Cre-LoxP strategy resulted in a null allele with Arl6ip5 loss-of-function in tissues from homozygous mutant mice. The phenotype analysis of Arl6ip5 $\mathrm{KO}$ mice revealed an unrecognized role of Arl6ip5 in bone metabolism. Specifically, gene ablation of Arl6ip5 in mice decreased bone mass owing to diminution of osteoblast differentiation and increase of osteoclast formation. Mechanistically, deficiency of Arl6ip5 appears to inhibit osteoblast proliferation and differentiation 


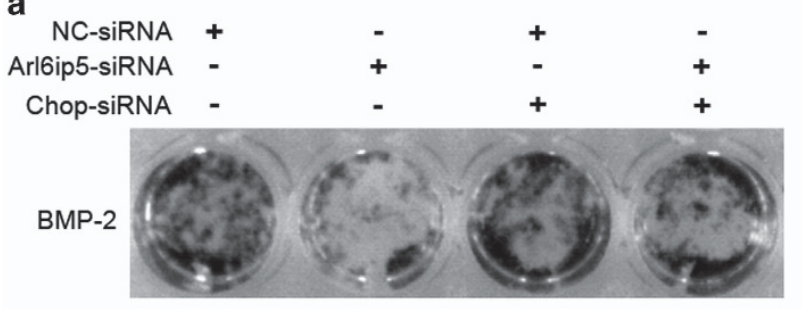

C

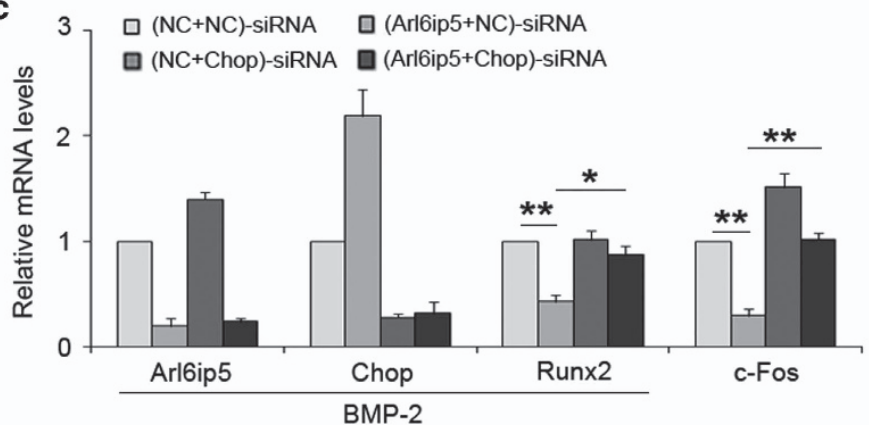

b

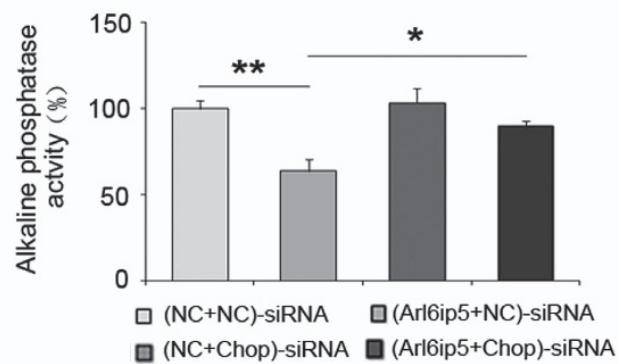

d

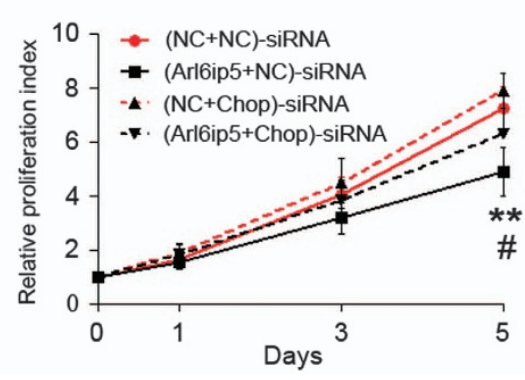

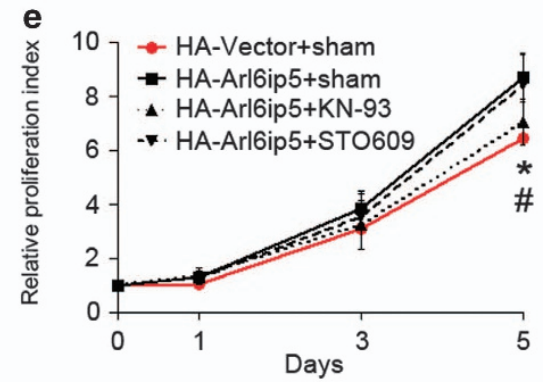

Figure 7 Chop involves in the regulation of Arl6ip5 on osteoblast proliferation and differentiation. (a-c) Double siRNA-treated UAMS-32 cells received with or without BMP-2 $(100 \mathrm{nM})$ treatment for 5 days to induced differentiation, then evaluated by ALP staining (a) and ALP activity analysis (b) and mRNA levels of Arl6ip5, Chop, Runx2 (c, with BMP-2 treatment) and c-Fos (c, without BMP-2 treatment). For the panels of ALP activity analysis and mRNA detection, ${ }^{*} P<0.01,{ }^{*} P<0.05$ based on ANOVA. (d) Cell proliferation in UAMS-32 cells with double siRNA treatment were analyzed with MTT assay. ${ }^{* *}(\mathrm{NC}+\mathrm{NC})$-siRNA versus (NC+Arl6ip5)-siRNA, $P<0.01 ;{ }^{\#}(\mathrm{Chop}+\mathrm{Arl6ip5})$-siRNA versus (NC + Arl6ip5)-siRNA, $P<0.05$. (e) Cell proliferation in HA-Arl6ip5 overexpressed UAMS-32 cells with or without KN-93 (1 $\mu \mathrm{M})$ and STO-609 (1 $\mu \mathrm{M})$ treatment were analyzed with

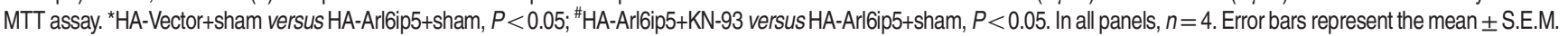

via the disturbance of calcium homeostasis and induction of apoptosis that mediated by ER stress, moreover, favor osteoclastogenesis through enhancing RANKL expression in osteoblast.

Arl6ip5, initially cloned from human tracheal bronchial epithelial cells after treatment with all-trans retinoic acid (ATRA), was regarded as a novel cell differentiationassociated gene, and regulated by differentiation inducers such as ATRA, phorbol-12-myristate-13-acetate, arabinoside and hemin. ${ }^{17,31,32}$ In human myeloid leukemia cells, Arl6ip5 was associated with ATRA-induced cellular differentiation and cellular proliferation. ${ }^{32}$ In neurite growth, GTRAP3-18, the rat homologue of Arl6ip5, was reported to be a negative regulator for neuronal differentiation. ${ }^{33}$ In this study, the expression of Arl6ip5 was increased during osteoblast differentiation. Moreover, Arl6ip5 mRNA level was induced by osteotropic factors, such as PTH, TGF- $\beta 1$, BMP-2 and Dex in UAMS-32 cells. Immunostaining of Arl6ip5 demonstrated that Arl6ip5 is an ER localized protein in osteoblast. These findings suggested that Arl6ip5 may be related to osteoblast proliferation and differentiation, which was supported by the findings that
Arl6ip5 deficiency in vitro impaired osteoblast proliferation and differentiation.

For the mechanisms underlying, we observed that Arl6ip5 regulated intracellular calcium level via its regulation on ER calcium channel that mediates calcium output from ER. It is important to note that the potential channels that is regulated by Arl6ip5 are different from the IP3R channel, as the IP3Rs inhibitor 2-APB only partially inhibited the increase of $\mathrm{Ca}^{2+}$ that is mediated by Arl6ip5 was overexpressed. Calcium signaling has been well demonstrated for its role in osteoblast proliferation and differentiation owing to its regulation on the expression and activity of the transcription factors, which are important for osteoblast differentiation, maturation and bone formation, for example, c-fos, Runx2 and osterix. ${ }^{10,34,35}$ It is plausible that the decrease of Runx2 and osterix was subsequent to the impaired calcium signaling in Arl6ip5 deficiency osteoblasts, although the activity of these transcription factors was not observed in this study. However, a recent study showed that mice with deficiency of CAMKK2, one upstream kinase of CaMKI and CaMKIV, possessed higher trabecular bone mass in their long bones and more OBs and 

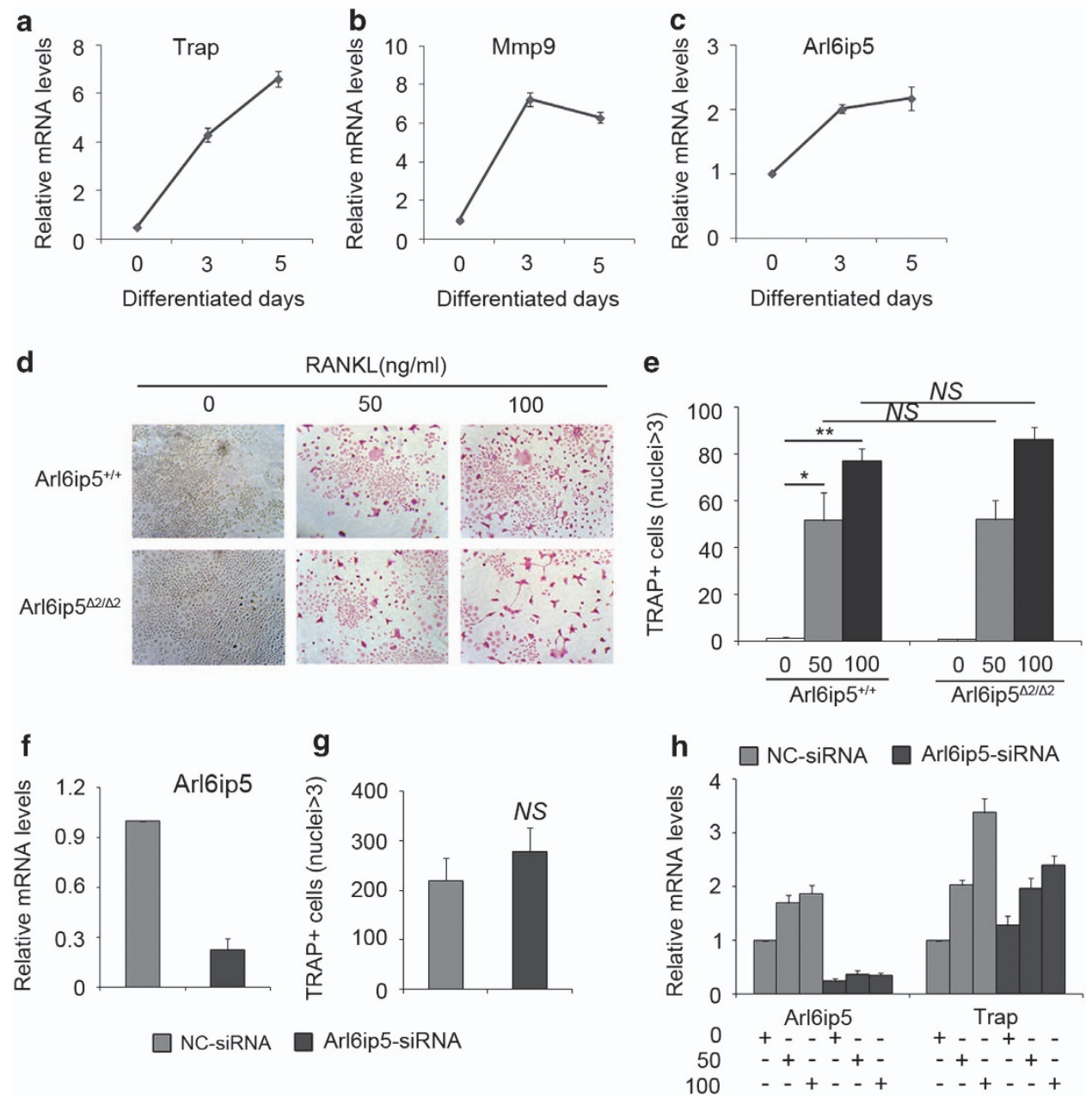

Figure 8 Arl6ip5 interference does not affect osteoclastogenesis. (a-c) BMMs were differentiated in the presence of $10 \mathrm{ng} / \mathrm{ml} \mathrm{M}-\mathrm{CSF}$ and $100 \mathrm{ng} / \mathrm{ml}$ RANKL for indicated days, then mRNA of Trap (a), Mmp9 (b) and Arl6ip5 (c) was detected, $n=3$. (d and e) BMMs from Arl6ip5 ${ }^{+/+}$and Arl6ip5 ${ }^{\Delta 2 / \Delta 2}$ mice were differentiated with $10 \mathrm{ng} / \mathrm{ml} \mathrm{M}-\mathrm{CSF}$ and 50 or $100 \mathrm{ng} / \mathrm{ml}$ RANKL for 6 days in 24 wells, then for TRAP staining. Representative fields were shown. Scale bars $=100 \mu \mathrm{m}$ (d). Histograms quantify the average numbers of multinucleated TRAP+ cells. Data represent mean \pm S.E.M. derived from three independent experiments, each using BMMs from three littermates (e). (f and $\mathbf{g}$ ) Raw264.7 cells received NC-siRNA or Arl6ip5siRNA were differentiated in the presence of 50 or $100 \mathrm{ng} / \mathrm{ml}$ RANKL for 5 days, then stained for TRAP activity and detected for the mRNA expression of Arl6ip5 and Trap. Histograms quantify the average numbers of multinucleated TRAP+ cells (f). Q-PCR verified the downregulation of Arl6ip5 (g). Q-PCR analyzed the mRNA expression of Arl6ip5 and Trap (h). $n=3$. Data represent mean \pm S.E.M. NS, nonsignificant. ${ }^{*} P<0.05 ;{ }^{*} P<0.01$

fewer multinuclear OCs. ${ }^{36}$ Whereas the activity of CaMKI and CaMKIV was not detected in this study, the activity of CaMKII, which is ubiquitously expressed and well studied in osteoblast differentiation, ${ }^{4,10,37}$ was decreased upon Arl6ip5 interference. As the activation of CaMKII by $\mathrm{Ca}^{2+} / \mathrm{CaM}$ binding and its autophosphorylation are CaMKK independent, ${ }^{38}$ it is difficult to evaluate the role of CaMKKs in the impairment of osteoblast differentiation owing to Arl6ip5 deficiency. However, for osteoblast proliferation, studies with inhibitor KN-93 (generous to CaMKI, CaMKIV and CaMKII) and STO-609 (specific for CaMKK and the downstream targets CaMKI and CaMKIV) showed that CaM-CaMKII but not CaMKK was involved for the regulation of Arl6ip5 on osteoblast proliferation. Further study with specific shRNA or siRNA for CaMKKs and CaMKs are warranted.

We also observed that Arl6ip5 deficiency induced ER stress, as indicated by the increase of ER stress-related genes. Mild ER stress is helpful for restoring cellular homeostasis, however, the persistent and unalleviated ER stress elicits apoptosis. ${ }^{12,16}$ In this study, the significant increase of Chop and its target gene Gadd34, two representative genes for the regulation of ER stress-mediated apoptosis, indicated the UPR to ER stress shift from pro-survival to pro-apoptosis in the cells with Arl6ip5 deficiency. The apoptosis was demonstrated by the in vivo and in vitro evidence that TUNEL-positive or Annexin-V-positive cells and the protein expression of cleave caspase-3 was increased in Arl6ip5 deficiency cells. Moreover, the increased processing of caspase-12, a welldemonstrated indicator for ER stress-induced apoptosis, ${ }^{39}$ and the reversed effect of chemical chaperon 4-PBA suggested that the apoptosis upon Arl6ip5 knocked-down is dependent on the ER stress.

IRE1- and Chop-mediated apoptosis pathway has a central role in ER stress-mediated apoptosis. ${ }^{16}$ In Arl6ip5 knockeddown osteoblast, the mRNA and protein levels of Chop were increased. However, the activity of IRE1 was comparable between Arl6ip5 knocked-down and normal expressed cells. Moreover, knocked-down Chop with specific siRNA reversed 


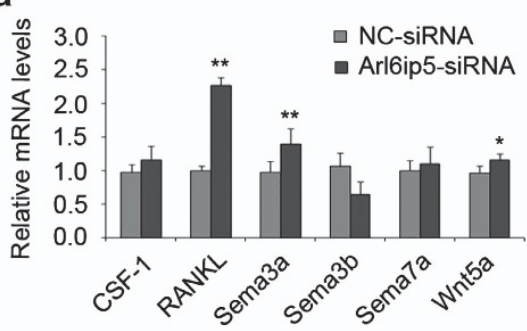

b

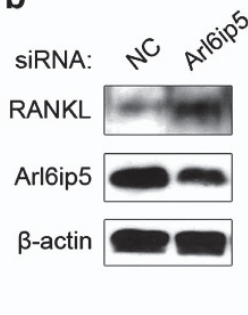

c

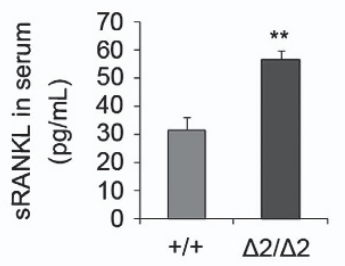

d

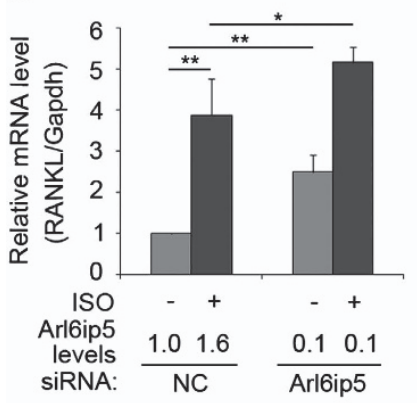

f
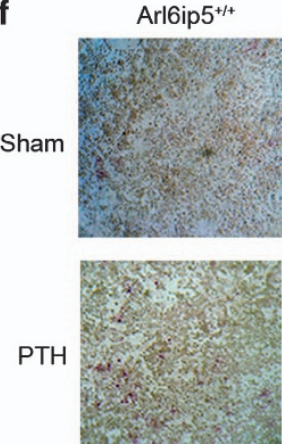

e

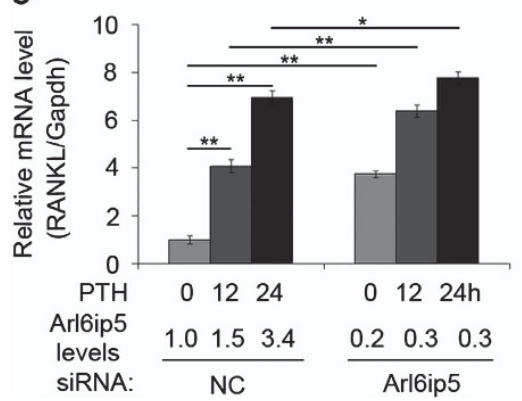

Arl6ip5 $5^{+/+}$
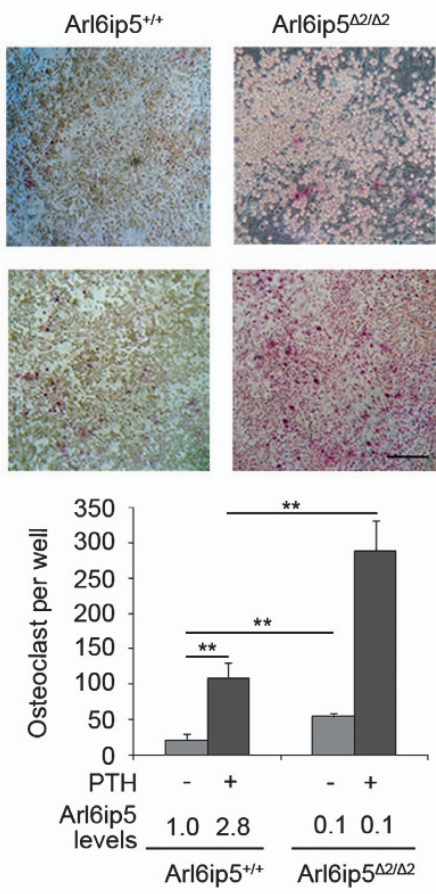

g

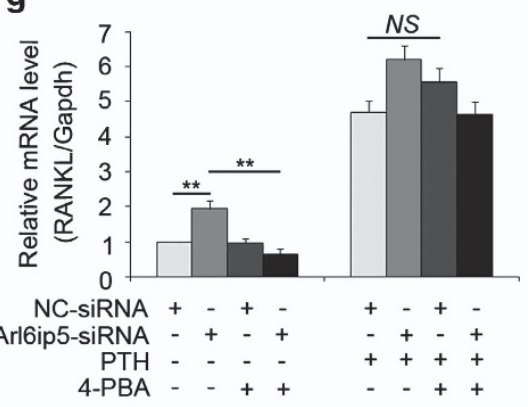

h

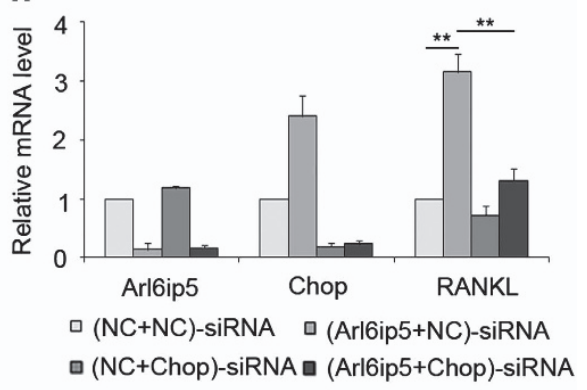

Figure 9 Osteoblastic Arl6ip5 deficiency enhances osteoclastogenesis via increasing RANKL expression. (a) Q-PCR was used to detect the expression of osteoblastosteoclast coupling factors. $n=6$. Data represent mean \pm S.E.M. ${ }^{*} P<0.05$ and ${ }^{\star \star} P<0.01$ by Students $t$-test. (b) Immunoblotting was used to measure the protein expression of RANKL, Arl6ip5 and $\beta$-actin in NC-siRNA and Arl6ip5-treated UAMS-32 cells. The densitometry results (arbitrary units) of three independent experiments were shown as a bar graph. Bar represents mean \pm S.E.M. ${ }^{*} P<0.05$ by Students $t$-test. (c) Serum was collected from 16-week-old Arl6ip5 ${ }^{\Delta 2 / \Delta 2}(n=6)$ and their wild-type littermates $\left(\right.$ Arl6ip5 $\left.{ }^{+/+}\right)$ $(n=6)$ and the sRANKL levels were analyzed with ELISA kit. Each bar represents mean \pm S.E.M. ${ }^{*} P<0.05$, Students $t$-test. (d and e) $Q$-PCR analyzed the RANKL transcription induced by ISO (d) and PTH (e) treatment in UAMS-32 cells with Arl6ip5-siRNA treatment. The mRNA level of Arl6ip5 was shown under the panel. (f) Osteoclast formation in cocultures of Arl6ip5 ${ }^{+/+}$, Arl6ip5 ${ }^{\Delta 2 / \Delta 2}$ POBs and bone marrow macrophages (BMMs) as osteoclast (OC) precursors, treated with or without PTH (100 nM). Representative images were shown in the upper panel. Scale bar $=1 \mathrm{~mm}$. Histograms quantify the average numbers of multinucleated TRAP+ cells. (g) UAMS-32 cells were treated with NC-siRNA and Arl6ip5-siRNA for $48 \mathrm{~h}$, then received PTH $(100 \mathrm{nM})$ with or without 4-PBA $(10 \mathrm{mM})$ for $24 \mathrm{~h}$. The transcription of RANKL was analyzed by Q-PCR. (h) UAMS-32 cells first received NC-siRNA or Chop-siRNA for $24 \mathrm{~h}$, then the medium was changed and further received NC-siRNA or Arl6ip5-siRNA for $72 \mathrm{~h}$. Q-PCR was used to analyze the mRNA levels of Chop, Arl6ip5 and RANKL. For $\mathbf{d}-\mathbf{h}$, bar represents mean \pm S.E.M. $(n=3) .{ }^{*} P<0.05$ and ${ }^{\star \star} P<0.01$ by ANOVA

the expression of its target genes and the apoptosis phenotype in Arl6ip5 knocked-down cells. These findings supported that Chop was involved in the regulation of apoptosis that mediated by Arl6ip5 interference. Chop regulates the expression of genes that related with apoptosis, ${ }^{40}$ such as Ero1, Bcl-2 and Trib3. We found that Trib3 was markedly increased but Ero1 and Bcl-2 expression was not changed in Arl6ip5 knocked-down cells. This differential may be cell type or stimulus dependent. Further study with gene expression array would be helpful for revealing the underlying mechanisms. Chop was shown to inhibit the differentiation of committed osteoblasts such as POBs but promote differentiation of ST-2, a multipotential mesenchymal progenitor cell line. ${ }^{27,28}$ In this study, knockeddown Chop increased the differention and proliferation of
Arl6ip5 deficiency UAMS-32 cells possibly via its regulation on the expression of Runx2 and c-Fos. Our results support the findings that Chop inhibits differentiation in committed osteoblast, as revealed by Shirakawa et al. ${ }^{28}$ UAMS-32 cells was isolated from mouse bone marrow cells and exhibited phenotypic characteristics of stromal/osteoblastic cells, ${ }^{41}$ but whether this cell line has multipotential property or just a committed osteoblst is still not clear. Further studies with Chop-specific deficiency in osteoblast on different stages (osteoblast progenitors, stromal marrow cells, pre-osteoblast and mature osteoblast) is important for the dual roles of Chop in osteoblast differentiation.

The in vivo study with Arl6ip5 $\mathrm{KO}$ mice indicated that the osteoclastogenesis was enhanced. However, the in vitro study with Arl6ip5 deficiency BMMs and Arl6ip5 knocked-down 
Raw264.7 cells showed that Arl6ip5 impaired in osteoclast progenitors had no effect on osteoclast formation, which prompted us to test the hypothesis that Arl6ip5 in osteoblasts have a critical role in osteoclastogenesis. We found that osteoblastic Arl6ip5 insufficiency significantly increased the expression of RANKL, the key factor for osteoclastogenesis. The subsequent in vitro study revealed the basal and stimuli (PTH, ISO and VitD3) induced RANKL gene expression and SRNAKL level were also significantly increased in Arl6ip5 knockdown osteoblast and Arl6ip5 KO POBs, moreover, the co-culture assay containing Arl6ip $5^{\Delta 2 / \Delta 2} \mathrm{POBs}$ with osteoclast precursors found more osteoclast formation and pit formation. These findings suggested that osteoblastic Arl6ip5 deficiency not only impaired osteoblast differentiation but also provided a microenvironment that suitable for osteoclastogenesis.

PTH and isoproterenol regulate RANKL expression in osteoblast via PKA-CREB and PKA-ATF4 pathway, respectively. ${ }^{42-44}$ However, it was the ER stress-related signaling but not the PKA-dependent pathway involved in the induction of basic RANKL transcription in Arl6ip5 knockeddown cells, as 4-PBA treatment but not $\mathrm{H}-89$ treatment blocked the effect of Arl6ip5 knocked-down on RANKL expression. The further study found that Chop, rather than ATF4, was the key factor to mediate the induction of basic RANKL expression in Arl6ip5 knocked-down cells. ATF4 has been demonstrated for its regulation on RANKL expression with ATF4 deficiency POBs, ${ }^{43}$ and ATF4 also regulates the genes that are important for alleviating ER stress. ${ }^{12}$ However, ATF4 interference by siRNA in this study, did not reduced the basic RANKL expression induced by ER stress as Arl6ip5 deficiency, indicated that ER stress mediates RANKL expression via ATF4-independent mechanisms. During ER stress, Chop also regulates the genes that involved in apoptosis and protein synthesis. ${ }^{40,45}$ It is still not clear whether the regulation mechanisms for these genes also implicated in the regulation of Chop on RANKL expression. Interestingly, the transgenic mice with Chop overexpressing showed impaired osteoblastic function and osteopenia owing to increased osteoblast apoptosis. ${ }^{46}$ Although further studies about the detailed mechanisms in the regulation of RANKL transcription are warranted, it is possible that the osteoclastogenic effect mediated by Chop found in this study also contributes to the osteopenia phenotype for Chop-overexpressing mice.

In conclusion, our results revealed a novel effect of osteoblastic Arl6ip5 in bone formation and signal couplings between osteoblast and osteoclast via its regulation on cellular homeostasis in bone metabolism. Arl6ip5 is an ER-resident protein and regulated by bone metabolism factors in osteoblast. Deficiency of Arl6ip5 in osteoblast disturbs the calcium homeostasis and induces ER stress-mediated apoptosis, then impairs osteoblast proliferation and differentiation, moreover, produces an osteoclastogenic microenvironment by the induction of RANKL. Therefore, the loss of Arl6ip5 disturbs the homeostasis of osteoblast and skeleton. However, the use of mice with Arl6ip5 global deficiency compromised the findings. Current efforts are focus on the cell lineage-specific conditional inactivation of the Arl6ip5 allele and the identification of Arl6ip5-interacting proteins in an attempt to gain further insights into the mechanism of activity and function of Arl6ip5 in skeletal disease.

\section{Materials and Methods}

Animals. Arl6ip5 KO mice (Arl6ip5 $5^{\Delta 2 / \Delta 2}$ ) were constructed as described before ${ }^{21}$ and kept in a pathogen-free environment in a standard breeding room. Mice used in this study were intercrossed for at least 10 generations and were maintained in a C57BL/6J background. Wild-type (Arl6ip5 ${ }^{+/+}$) littermates were used as controls. Mouse experiments were approved by the Jiangsu Institute of Nuclear Medicine Animal Ethical Committee and conducted in accordance with Animal Care and Use Committee of the Model Animal Research Centre.

Reagents and antibodies. Fetal bovine serum (FBS), L-glutamine, antibiotics, alpha-modified essential medium ( $\alpha$-MEM) and trypsin/EDTA were obtained from Gibco (Life Technologies, Grand Island, NY, USA). PTH, Dex, 1,25$(\mathrm{OH})_{2} \mathrm{D}_{3}, \mathrm{MTT}$, DMSO, ascorbic acid, $\beta$-glycerophosphate, indomethacin, TG, 2-APB, 4-PBA, KN-93 and STO-609 were purchased from Sigma (Sigma-Aldrich, St. Louis, MO, USA). ISO was from TCI (Tokyo Chemical Industry, Tokyo, Japan). TGF $\beta 1$, BMP-2 and RANKL were from R\&D (R\&D Systems, Inc., Minneapolis, MN, USA). Type 2 collagenase was obtained from Worthington (Worthington Biochemical Corporation, Lakewood, NJ, USA). The antibodies for CaM, p-CaMKII and CaMKII were from Epitomics (Epitomics-an Abcam Company, Burlingame, CA, USA). Caspase-3 and caspase-12 antibodies from Cell Signaling (Cell Signaling Technology, Inc., Danvers, MA, USA). p-IRE1 (Ser724) and IRE1 antibodies were from Novus (Novus Biologicals, LLC, Littleton, CO, USA). Chop antibody from Beyotime (Beyotime Institute of Biotechnology, Nantong, China). Anti-HA and $\beta$-actin antibodies were from Santa Cruz (Santa Cruz Biotechnology, Inc., Santa Cruz, CA, USA). Arl6ip5 antibody was used as described before.

Cells culture. UAMS-32 stromal/osteoblastic cell line was a gift from Professor Charles A O'Brien ${ }^{41}$ (University of Arkansas for Medical Sciences, Little Rock, AR, USA) and maintained in $\alpha$-MEM containing 10\% FBS, 2 mM L-glutamine and antibiotics. POBs were extracted from both genders of newborn neonatal mice calvaria with sequential type 2 collagenase digestion and cultured in $\alpha$-MEM containing $15 \%$ FBS, $2 \mathrm{mM}$ L-glutamine and antibiotics. The POBs used in this study were passaged 2-4 times. Raw264.7 cell were purchased from ATCC (American Type Culture Collection, Manassas, VA , USA) and maintained in $\alpha$-MEM containing 10\% FBS and antibiotics. For inducing POB differentiation, cells were cultured in medium containing $50 \mu \mathrm{M}$ ascorbic acid, $10 \mathrm{mM} \beta$-glycerophosphate, $100 \mu \mathrm{M}$ indomethacin and $100 \mathrm{nM}$ Dex for the indicated time with regular medium changed. Non-adherent bone marrow cells were isolated from femurs and tibia of 60- to 90-day-old C57BL/6J mice and the suspended cells were collected after 48-h culture were used for co-culture assay. The UAMS-32 cells were treated with BMP-2 $(100 \mathrm{ng} / \mathrm{ml})$ and the Raw264.7 cells were treated with RANKL $(100 \mathrm{ng} / \mathrm{ml})$ to induce differentiation.

Plasmids and siRNA. cDNA extracted from UAMS-32 cells was used. Full-length Arl6ip5 with $\mathrm{HA}$ tagged on $\mathrm{C}$ terminus (HA-Arl6ip5) was constructed with the primers (from $5^{\prime}$ to $3^{\prime}$ ): sense - CGCGGATCCGCCACCATGGACGTGAACCTC GCC; antisense - CGGTCTAGATTAAGCGTAGTCTGGGACGTCGTATGGGTACT CCCTCGCTTTGCTGATGTA and inserted into pCDNA-3.1(+) vector with BamH and Xbal. Full-length Arl6ip5 with EGFP tagged on C terminus (Arl6ip5-EGFP) was constructed with the primers (from $5^{\prime}$ to $3^{\prime}$ ): sense - GCGGAATTCATGGACGTGAA CCTCG; antisense - GGTGGATCCCTCCCTCGCTTTGCTG and inserted into EGFP-N1 vector with EcoRI and BamHI. The plasmids were verified by sequencing. Plasmids were transfected into UAMS-32 cells or POB with Lipofectamine 2000. UAMS-32 cells with stable plasmid expression were selected under $100 \mu \mathrm{g} / \mathrm{ml}$ Geneticin (Sigma-Aldrich)

siRNA specific for Arl6ip5 and negative control siRNA were purchased from siGENOME SMART pool (Thermo Fisher Scientific, Rockford, IL, USA) and transfected using Lipofectamine 2000 (Life Technologies) according to the manufacturer's protocol. The sequence of siRNA is as below $\left(5^{\prime}\right.$ to $\left.3^{\prime}\right)$ : UCUAUUACCAGACCAACAU. The siRNA for Chop and ATF4 was purchased from Ruibo (Guangzhou RiboBio Co., Ltd., Guangzhou, China). For double siRNA transfection, UAMS-32 cells first received NC-siRNA or Chop-siRNA or ATF4-siRNA for $24 \mathrm{~h}$, then the medium was changed and further received NC-siRNA or Arl6ip5siRNA for $72 \mathrm{~h}$. The efficiency of siRNA treatments were evaluated by quantitative polymerase chain reaction (Q-PCR) or immunoblotting as indicated.

Q-PCR analysis. The mRNA extracted from cells or tissues was first reverse transcribed to cDNA with High Capacity cDNA Reverse Transcription Kit (Applied 
Biosystems, Carlsbad, CA, USA), then analyzed on ABI Prism 7500 sequence detection system with SYBR Premix Ex Taq Mix (TaKaRa Bio Inc., Otsu, Japan) for the expression of Arl6ip5, alkaline phosphatase, osteopontin, Trap, Ctsk, Mmp9, Bip, Chop, Grp94, Pdia3, P4hb, Gadd34, Trib3, c-Fos, $\beta$-actin and Gapdh, the primers for these genes were retrieved from PrimerBank. ${ }^{47}$ For mRNA expression of osterix (Mm04209856_m1), Runx2 (Mm00501580_m1), osteocalcin (Mm03413826_mH) and RANKL (Mm00441908_m1), the Taqman primers and probes indicated were used. All detections were in triplicate for each sample and data were normalized to Gapdh or $\beta$-actin levels $\left({ }^{\Delta \Delta} \mathrm{CT}\right)$.

Proliferation and apoptosis analysis. The cell proliferation was detected with MTT assay as previously indicated with some in-house modifications. ${ }^{48}$ Briefly, $1 \times 10^{3}$ UAMS-32 cells with Arl6ip5 stable expression or $1 \times 10^{3}$ UAMS-32 cells received the siRNA treatment for the indicated days or $2 \times 10^{3}$ POBs were seeded on 96-well microplate, cultured with or without KN-93 $(1 \mu \mathrm{M})$ and STO-609 $(1 \mu \mathrm{M})$ treatment for indicated days and then $2 \mathrm{mM}$ MTT was added into the cultured medium in $1: 10$ (vol:vol) ratio and incubated in $37^{\circ} \mathrm{C}$ for $2 \mathrm{~h}$, then the medium was replaced with DMSO and the plate was continuously incubated in $37^{\circ} \mathrm{C}$ for another half hour in the dark and the absorbance of colored solution at $470 \mathrm{~nm}$ was measured by SpetraMax M5 microplate reader (Molecular Devices, Sunnyvale, CA, USA). For the proliferation analysis of double-siRNAtreated UAMS-32 cells, $24 \mathrm{~h}$ after the second siRNA treatment, cells were trypsinized and seeded into 96-well microplate for MTT analysis. Apoptosis analysis with Annexin-V-FLUOS Staining Kit (Roche Applied Science, Indianapolis, IN, USA) and DeadEnd Colorimetric TUNEL System (Promega, Madison, WI, USA) followed the protocol provided.

ALP staining and activity measurement. At first, $1 \times 10^{4} \mathrm{POBs}$ per well in 24-well culture plates were cultured and differentiated in $\alpha$-MEM medium containing $50 \mu \mathrm{M}$ ascorbic acid, $10 \mathrm{mM} \beta$-glycerophosphate, $100 \mu \mathrm{M}$ indomethacin and $100 \mathrm{nM}$ Dex for indicated time with regular medium changed. TRACP and ALP double-staining Kit (TaKaRa Bio Inc.) was used for ALP staining. Alkaline Phosphatase Assay Kit (Beyotime Institute of Biotechnology) was used for ALP activity analysis.

Western blotting. For western blotting, total cytoplasmic protein was isolated with RIPA lysis buffer containing protease and phosphatase inhibitors (Halt Protease Inhibitor Cocktail) from Pierce (Thermo Fisher Scientific Inc.) and run on 7.5-12\% SDS-PAGE gels. After blotting to PVDF membrane (Life Technologies), membranes were blocked for $60 \mathrm{~min}$ at room temperature and incubated overnight at $4{ }^{\circ} \mathrm{C}$ in buffer (TBS with $0.1 \%$ Tween 20 and $5 \%$ non-fat milk powder) containing diluted antibodies. Detection of primary antibodies was performed with HRP-conjugated secondary antibody. Immunoreactive bands were visualized with Chemiluminescent substrate (Thermo Fisher Scientific Inc.). Densitometric analysis was performed using Image J software (National Institutes of Health, Bethesda, MD, USA).

ELISA and immunostaining. sRNAKL in culture medium or in serum was detected with mouse RANKL ELISA kit from R\&D (R\&D Systems, Inc.) following the manufacturer's protocol. C-telopeptide of Collagen alpha-1(II) chain (CTX-II) in mice serum was detected with ELISA Kit for Mouse C-telopeptide of Collagen alpha-1(II) chain from ElAab (Wuhan ElAab Science Co. Ltd., Wuhan, China). For immunofluorescence staining, cells with Arl6ip5-EGFP overexpressed were incubated with ER-tracker from Molecular Probe (Life Technologies) for 0.5 to $2 \mathrm{~h}$ as indicated by the protocols. For the endogenous Arl6ip5, cells were fixed in methanol and incubated with the PBST containing the diluted primary antibodies for Arl6ip5 and calnexin overnight. Secondary antibodies were goat anti-rabbit or antimouse antibody conjugated to Alexa Fluor 488 or 594 from Molecular Probe (Life Technologies). Images were acquired with Olympus Laser Scanning Confocal Microscope (Olympus Corporation, Tokyo, Japan). Image $\mathrm{J}$ software was used to merge images.

$\left[\mathrm{Ca}^{2+}\right]_{\mathrm{i}}$ measurements. The $\left[\mathrm{Ca}^{2+}\right]_{i}$ of UAMS-32 cells with stable expression of either HA-Arl6ip5 or control vector and cells transiently transfected with Arl6ip5siRNA (Thermo Fisher Scientific Inc.) were measured by loading with $5 \mu \mathrm{M}$ Fura2AM (Sigma-Aldrich) prepared in extracellular buffer with no calcium (125 mM NaCl, $5 \mathrm{mM} \mathrm{KCl}, 1.5 \mathrm{mM} \mathrm{MgCl}_{2}, 20 \mathrm{mM}$ HEPES, $10 \mathrm{mM}$ glucose, pH7.4) for 30 min at $37^{\circ}$ C. For the stimulation, $200 \mu \mathrm{M}$ of ATP was added to the cells and the $\mathrm{Ca}^{2+}$ transients were recorded as the $340 / 380 \mathrm{~nm}$ ratio $(R)$ of the resulting $510-\mathrm{nm}$ emissions using SpetraMax plate reader (Molecular Devices). For inhibition experiments, cells were incubated for $30 \mathrm{~min}$ before analysis with one of the following inhibitors: $100 \mu \mathrm{M}$ 2-APB for blocking IP3Rs, $0.5 \mu \mathrm{M}$ TG for SERCA ER $\mathrm{Ca}^{2+}$ pump inactivation. The $\left[\mathrm{Ca}^{2+}\right]_{i}$ levels were calculated as described previously using the equation $\left[\mathrm{Ca}^{2+}\right]_{i}=K d^{*}\left(\mathrm{R}-\mathrm{R}_{\min }\right) /\left(\mathrm{R}_{\max }-\mathrm{R}\right)\left(\mathrm{F} 380_{\max } / \mathrm{F} 380_{\min }\right),{ }^{49}$ where $\mathrm{R}_{\min }$ is the ratio at zero $\mathrm{Ca}^{2+}, \mathrm{R}_{\max }$ is the ratio when Fura-2 is completely saturated with $\mathrm{Ca}^{2+}, \mathrm{F} 380$ min is the fluorescence at $380 \mathrm{~nm}$ for zero $\mathrm{Ca}^{2+}$ and $\mathrm{F} 380_{\max }$ is the fluorescence at saturating $\mathrm{Ca}^{2+}$ and $\mathrm{Kd}=224 \mathrm{nM}$.

Osteoclast formation assay and pit formation assay. Calvarial cells (POBs) isolated from neonatal Arl6ip5 $5^{\Delta 2 / \Delta 2}$ or wild-type mice were co-cultured with non-adherent bone marrow cells. POBs at a density of $1 \times 10^{3}$ cells per well in 48 well plates were co-cultured with $2 \times 10^{4}$ per well non-adherent bone marrow cells isolated from wild-type mice for 7-9 days in $\alpha$-MEM containing 10\% FBS and $100 \mathrm{nM}$ PTH. One-half of the medium was replaced with fresh medium and PTH every 3 days. After 9 days co-cultures, cells were fixed and stained for TRAP using TRACP and ALP double-stain Kit (TaKaRa Bio Inc.). The osteoclast with three or more nucleus was calculated with Image $\mathrm{J}$ software. For resorption analysis, $1 \times 10^{4}$ Raw264.7 cells were seeded into the 24-well culture plate that contained $2 \times 10^{3}$ POBs and dentine slices (Immunodiagnostic Systems PLC, Boldon, UK), $100 \mathrm{nM}$ PTH was added the next day and culture for 5-6 days with regular medium changed. Slices were incubated with $0.25 \mathrm{M}$ ammonium hydroxide and sonicated for several times. The slices were stained with $0.1 \%$ toluidine blue in $0.5 \%$ sodium tetraborate for $5 \mathrm{~min}$, washed with water and air dried before photographs were taken by reflected light microscope. The resorpted areas were measured by Image J software.

Bone histomorphometric analysis. For bone histomorphometric analysis, 4-month-old Arl6ip5 KO mice (Arl6ip5 ${ }^{\Delta 2 / \Delta 2}$ ) of both genders and their wild-type littermates were used. $\mu \mathrm{CT}$ and bone histomorphometry were carried out as previously described with slight modification. ${ }^{50}$ Briefly, for $\mu$-CT analysis, tibia isolated from each mouse were scanned by using a cone-beam microfocus $\mathrm{X}$-ray computed tomography ( $\mu \mathrm{CT} 40$; Scanco Medical AG, Brüttisellen, Switzerland), image acquisition was performed at $100 \mathrm{kV}$ and $98 \mu \mathrm{A}$ with a 0.9-degree rotation between frames. The resolution of the $\mu \mathrm{CT}$ images is $18.2 \mu \mathrm{m}$. For dynamic bone histomorphometry, double calcein ( $10 \mu \mathrm{g} / \mathrm{g})$ (C-0875, Sigma, St. Louis, MO, USA) were intraperitoneally injected on 15 and 5 days mice (4-month-old Arl6ip5 $5^{\Delta 2 / \Delta 2}$ and their wild-type littermates) before they were killed. Then the tibias were harvested and embedded in methyl methacrylate. Serial sections were cut, viewed and imaged using fluorescence microscopy (Olympus IX53 microscope). The double calceinlabeled width of endosteum of cortical bone and trabecular bone was measured and the MAR was calculated as the interlabel width/labeling period. For static bone histomorphometry, tibias were fixed in $4 \%$ neutral buffered formalin, decalcified in EDTA ( $\mathrm{pH} 7.2)$, embedded in paraffin and sectioned at a thickness of $5 \mu \mathrm{m}$. The sections were stained with hematoxylin and eosin staining and TRAP staining and the number of osteoblast and osteoclast in the cortical bone of metaphysis were measured.

Statistical analysis. Results are expressed as the mean \pm S.E.M. Statistical significance was identified by Student's $t$-test or ANOVA where appropriate, with probability $P<0.05$ being considered significant.

\section{Conflict of Interest}

The authors declare no conflict of interest.

Acknowledgements. We thank Dr. Oluf Dimitri Røe of the Department of Cancer Research and Molecular Medicine, Norwegian University of Science and Technology for his careful and critical editing for the language. This work was supported in part by grants from the NSFC $(81200645,30930080)$, Postdoctoral Fund of Jiangsu Province, the Ministry of Health of China Grant (LW201006) and the Department of Science and Technology of Jiangsu Province Grant (SB201077641).

1. Boyle WJ, Simonet WS, Lacey DL. Osteoclast differentiation and activation. Nature 2003; 423: 337-342.

2. Long F. Building strong bones: molecular regulation of the osteoblast lineage. Nat Rev $\mathrm{Mol}$ Cell Biol 2011; 13: 27-38.

3. Harada S, Rodan GA. Control of osteoblast function and regulation of bone mass. Nature 2003; 423: 349-355. 
4. Zayzafoon M. Calcium/calmodulin signaling controls osteoblast growth and differentiation J Cell Biochem 2006; 97: 56-70.

5. Saito A, Ochiai K, Kondo S, Tsumagari K, Murakami T, Cavener DR et al. Endoplasmic reticulum stress response mediated by the PERK-elF2(alpha)-ATF4 pathway is involved in osteoblast differentiation induced by BMP2. J Biol Chem 2011; 286: 4809-4818.

6. Moore KA, Hollien J. The unfolded protein response in secretory cell function. Annu Rev Genet 2012; 46: 165-183.

7. Clapham DE. Calcium signaling. Cell 2007; 131: 1047-1058.

8. Berridge MJ, Lipp P, Bootman MD. The versatility and universality of calcium signalling. Nat Rev Mol Cell Biol 2000; 1: 11-21.

9. Patterson RL, Boehning D, Snyder SH. Inositol 1,4,5-trisphosphate receptors as signal integrators. Annu Rev Biochem 2004; 73: 437-465.

10. Zayzafoon M, Fulzele K, McDonald JM. Calmodulin and calmodulin-dependent kinase Ilalpha regulate osteoblast differentiation by controlling c-fos expression. J Biol Chem 2005; 280: 7049-7059.

11. Mekahli D, Bultynck G, Parys JB, De Smedt H, Missiaen L. Endoplasmic-reticulum calcium depletion and disease. Cold Spring Harb Perspect Biol 2011; 3: a004317.

12. Walter $P$, Ron $D$. The unfolded protein response: from stress pathway to homeostatic regulation. Science 2011; 334: 1081-1086.

13. Murakami T, Saito A, Hino S, Kondo S, Kanemoto S, Chihara K et al. Signalling mediated by the endoplasmic reticulum stress transducer OASIS is involved in bone formation. Nat Cell Biol 2009; 11: 1205-1211.

14. Yang X, Matsuda K, Bialek $P$, Jacquot $S$, Masuoka HC, Schinke T et al. ATF4 is a substrate of RSK2 and an essential regulator of osteoblast biology; implication for Coffin-Lowry Syndrome. Cell 2004; 117: 387-398.

15. Hetz $\mathrm{C}$. The unfolded protein response: controlling cell fate decisions under ER stress and beyond. Nat Rev Mol Cell Biol 2012; 13: 89-102

16. Tabas I, Ron D. Integrating the mechanisms of apoptosis induced by endoplasmic reticulum stress. Nat Cell Biol 2011; 13: 184-190.

17. Lin $\mathrm{Cl}$, Orlov I, Ruggiero AM, Dykes-Hoberg M, Lee A, Jackson M et al. Modulation of the neuronal glutamate transporter EAAC1 by the interacting protein GTRAP3-18. Nature 2001; 410: 84-88.

18. Mao WG, Li AP, Ye J, Huang S, Li AQ, Zhou JW. [Effect of differentiation inducer and heat stress on the expression of JWA protein and Hsp70 of K562 cells]. Zhonghua Lao Dong Wei Sheng Zhi Ye Bing Za Zhi 2003; 21: 253-256.

19. Ruggiero AM, Liu Y, Vidensky S, Maier S, Jung E, Farhan $\mathrm{H}$ et al. The endoplasmic reticulum exit of glutamate transporter is regulated by the inducible mammalian Yip6b/ GTRAP3-18 protein. J Biol Chem 2008; 283: 6175-6183.

20. Allan EH, Hausler KD, Wei T, Gooi JH, Quinn JM, Crimeen-Irwin B et al. EphrinB2 regulation by PTH and PTHrP revealed by molecular profiling in differentiating osteoblasts. $J$ Bone Miner Res 2008; 23: 1170-1181.

21. Gong Z, Shi Y, Zhu Z, Li X, Ye Y, Zhang J et al. JWA deficiency suppresses dimethylbenz[a] anthracene-phorbol ester induced skin papillomas via inactivation of MAPK pathway in mice. PLoS One 2012; 7: e34154.

22. Ishikawa M, Iwamoto T, Nakamura T, Doyle A, Fukumoto S, Yamada Y. Pannexin 3 functions as an $\mathrm{ER} \mathrm{Ca}(2+)$ channel, hemichannel, and gap junction to promote osteoblas differentiation. J Cell Biol 2011; 193: 1257-1274

23. Brandman O, Liou J, Park WS, Meyer T. STIM2 is a feedback regulator that stabilizes basa cytosolic and endoplasmic reticulum Ca2+ levels. Cell 2007; 131: 1327-1339.

24. Maruyama T, Kanaji T, Nakade S, Kanno T, Mikoshiba K. 2APB, 2-aminoethoxydipheny borate, a membrane-penetrable modulator of Ins(1,4,5)P3-induced Ca2+ release. J Biochem 1997; 122: 498-505

25. Treiman M, Caspersen C, Christensen SB. A tool coming of age: thapsigargin as an inhibitor of sarco-endoplasmic reticulum Ca(2+)-ATPases. Trends Pharmacol Sci 1998; 19 131-135.

26. Ohoka N, Yoshii S, Hattori T, Onozaki K, Hayashi H. TRB3, a novel ER stress-inducible gene is induced via ATF4-CHOP pathway and is involved in cell death. EMBO J 2005; 24 1243-1255.

27. Pereira RC, Delany AM, Canalis E. CCAAT/enhancer binding protein homologous protein (DDIT3) induces osteoblastic cell differentiation. Endocrinology 2004; 145: 1952-1960.

28. Shirakawa K, Maeda S, Gotoh T, Hayashi M, Shinomiya K, Ehata S et al. CCAAT/enhancerbinding protein homologous protein (CHOP) regulates osteoblast differentiation. Mol Cell Bio 2006; 26: 6105-6116.

29. Wu Y, Sun H, Song F, Fu D, Wang J. DDIT3 overexpression increases odontoblastic potential of human dental pulp cells. Cell Prolif 2014; 47: 249-257.

30. Sims NA, Martin TJ. Coupling the activities of bone formation and resorption: a multitude of signals within the basic multicellular unit. Bonekey Rep 2014; 3: 481.

31. Zhu T, Chen R, Li AP, Liu J, Liu QZ, Chang HC et al. Regulation of a novel cell differentiation-associated gene, JWA during oxidative damage in K562 and MCF-7 cells. J Biomed Sci 2005; 12: 219-227.
32. Huang S, Shen Q, Mao WG, Li AP, Ye J, Liu QZ et al. JWA, a novel signaling molecule, involved in all-trans retinoic acid induced differentiation of HL-60 cells. J Biomed Sci 2006; 13: $357-371$.

33. Maier S, Reiterer V, Ruggiero AM, Rothstein JD, Thomas S, Dahm R et al. GTRAP3-18 serves as a negative regulator of Rab1 in protein transport and neuronal differentiation. J Cell Mol Med 2009; 13: 114-124.

34. Choi YH, Choi JH, Oh JW, Lee KY. Calmodulin-dependent kinase II regulates osteoblast differentiation through regulation of osterix. Biochem Biophys Res Commun 2013; 432: 248-255.

35. Eapen A, Kulkarni R, Ravindran S, Ramachandran A, Sundivakkam P, Tiruppathi C et al. Dentin phosphophoryn activates Smad protein signaling through $\mathrm{Ca2+-calmodulin-}$ dependent protein kinase II in undifferentiated mesenchymal cells. J Biol Chem 2013; 288 . 8585-8595.

36. Cary RL, Waddell S, Racioppi L, Long F, Novack DV, Voor MJ et al. Inhibition of $\mathrm{Ca}(2)$ $(+) /$ calmodulin-dependent protein kinase kinase 2 stimulates osteoblast formation and inhibits osteoclast differentiation. J Bone Miner Res 2013; 28: 1599-1610.

37. Seo JH, Jin YH, Jeong HM, Kim YJ, Jeong HG, Yeo CY et al. Calmodulin-dependent kinase II regulates Dlx5 during osteoblast differentiation. Biochem Biophys Res Commun 2009; 384: 100-104.

38. Means AR. The year in basic science: calmodulin kinase cascades. Mol Endocrino/ 2008; 22: 2759-2765.

39. Nakagawa T, Zhu H, Morishima N, Li E, Xu J, Yankner BA et al. Caspase-12 mediates endoplasmic-reticulum-specific apoptosis and cytotoxicity by amyloid-beta. Nature 2000; 403: $98-103$

40. Sano R, Reed JC. ER stress-induced cell death mechanisms. Biochim Biophys Acta 2013; 1833: $3460-3470$

41. O'Brien CA, Gubrij I, Lin SC, Saylors RL, Manolagas SC. STAT3 activation in stromal/ osteoblastic cells is required for induction of the receptor activator of NF-kappaB ligand and stimulation of osteoclastogenesis by gp130-utilizing cytokines or interleukin-1 but not 1,25-dihydroxyvitamin D3 or parathyroid hormone. J Biol Chem 1999; 274 : 19301-19308.

42. Fu Q, Jilka RL, Manolagas SC, O'Brien CA. Parathyroid hormone stimulates receptor activator of NFkappa B ligand and inhibits osteoprotegerin expression via protein kinase A activation of cAMP-response element-binding protein. J Biol Chem 2002; 277 : 48868-48875.

43. Elefteriou F, Ahn JD, Takeda S, Starbuck M, Yang X, Liu X et al. Leptin regulation of bone resorption by the sympathetic nervous system and CART. Nature 2005; 434: 514-520.

44. Fu Q, Manolagas SC, O'Brien CA. Parathyroid hormone controls receptor activator of NF-kappaB ligand gene expression via a distant transcriptional enhancer. Mol Cell Biol 2006; 26: $6453-6468$

45. Han J, Back SH, Hur J, Lin YH, Gildersleeve R, Shan J et al. ER-stress-induced transcriptional regulation increases protein synthesis leading to cell death. Nat Cell Biol 2013; 15: 481-490

46. Pereira RC, Stadmeyer LE, Smith DL, Rydziel S, Canalis E. CCAAT/Enhancer-binding protein homologous protein (CHOP) decreases bone formation and causes osteopenia. Bone 2007; 40: 619-626.

47. Wang X, Spandidos A, Wang H, Seed B. PrimerBank: a PCR primer database for quantitative gene expression analysis, 2012 update. Nucleic Acids Res 2012; 40: D1144-D1149.

48. Mao WG, Liu ZL, Chen R, Li AP, Zhou JW. JWA is required for the antiproliferative and pro-apoptotic effects of all-trans retinoic acid in Hela cells. Clin Exp Pharmacol Physiol 2006; 33: $816-824$.

49. Takahashi A, Camacho P, Lechleiter JD, Herman B. Measurement of intracellular calcium. Physiol Rev 1999; 79: 1089-1125.

50. Zhang HW, Ding J, Jin JL, Guo J, Liu JN, Karaplis A et al. Defects in mesenchymal stem cell self-renewal and cell fate determination lead to an osteopenic phenotype in Bmi-1 null mice. J Bone Miner Res 2010; 25: 640-652.

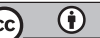

Cell Death and Disease is an open-access journal published by Nature Publishing Group. This work is licensed under a Creative Commons Attribution 4.0 International Licence. The images or other third party material in this article are included in the article's Creative Commons licence, unless indicated otherwise in the credit line; if the material is not included under the Creative Commons licence, users will need to obtain permission from the licence holder to reproduce the material. To view a copy of this licence, visit http://creativecommons.org/licenses/by/4.0 